\title{
ADAM17 substrate release in proximal tubule drives kidney fibrosis
}

Eirini Kefaloyianni, ${ }^{1}$ Muthu Lakshmi Muthu, ${ }^{1}$ Jakob Kaeppler, ${ }^{1}$ Xiaoming Sun, ${ }^{1}$ Venkata Sabbisetti, ${ }^{1}$ Athena Chalaris, ${ }^{2}$ Stefan Rose-John, ${ }^{2}$ Eitan Wong, ${ }^{3}$ Irit Sagi, ${ }^{3}$ Sushrut S. Waikar, ${ }^{1}$ Helmut Rennke, ${ }^{1}$ Benjamin D. Humphreys, ${ }^{4}$ Joseph V. Bonventre, ${ }^{1}$ and Andreas Herrlich ${ }^{1}$

${ }^{1}$ Renal Division, Brigham and Women's Hospital, Boston, Massachusetts, USA. ${ }^{2}$ Institute for Biochemistry, Christian-Albrechts-Universität zu Kiel, Kiel, Germany. ${ }^{3}$ Weizmann Institute of Science, Rehovot, Israel. ${ }^{4}$ Division of Nephrology, Washington University School of Medicine, St. Louis, Missouri, USA.

Kidney fibrosis following kidney injury is an unresolved health problem and causes significant morbidity and mortality worldwide. In a study into its molecular mechanism, we identified essential causative features. Acute or chronic kidney injury causes sustained elevation of a disintegrin and metalloprotease 17 (ADAM17); of its cleavage-activated proligand substrates, in particular of pro-TNF $\alpha$ and the EGFR ligand amphiregulin (pro-AREC); and of the substrates' receptors. As a consequence, EGFR is persistently activated and triggers the synthesis and release of proinflammatory and profibrotic factors, resulting in macrophage/neutrophil ingress and fibrosis. ADAM17 hypomorphic mice, specific ADAM17 inhibitor-treated WT mice, or mice with inducible KO of ADAM17 in proximal tubule (SIc34a1-Cre) were significantly protected against these effects. In vitro, in proximal tubule cells, we show that AREG has unique profibrotic actions that are potentiated by TNF $\alpha$-induced AREG cleavage. In vivo, in acute kidney injury (AKI) and chronic kidney disease (CKD, fibrosis) patients, soluble AREG is indeed highly upregulated in human urine, and both ADAM17 and AREC expression show strong positive correlation with fibrosis markers in related kidney biopsies. Our results indicate that targeting of the ADAM17 pathway represents a therapeutic target for human kidney fibrosis.

Conflict of interest: The authors have declared that no conflict of interest exists.

Submitted: February 12, 2016 Accepted: July 12, 2016 Published: August 18, 2016

Reference information: JCl Insight. 2016;1(13):e87023. doi:10.1172/jici.insight.87023.

\section{Introduction}

Kidney fibrosis (chronic kidney disease, CKD) is common and confers a remarkably high morbidity and mortality. It can result from a variety of injuries and diseases, very commonly diabetes and hypertension. Acute kidney injury (AKI) occurs in up to $10 \%-20 \%$ of adult patients hospitalized with acute illness, is associated with significant mortality (15\%-30\%; up to $70 \%$ if multiorgan failure in ICU patients), and causes progressive kidney fibrosis in survivors $(1,2)$. Regardless of the initial insult, CKD is characterized by progressive nephron loss involving glomerulosclerosis and tubulointerstitial fibrosis, leading to end stage renal disease (ESRD) and need for dialysis. Mortality on dialysis is roughly 30\% per year; however, specific therapies to prevent, slow down, or reverse fibrosis are severely lacking (3).

Over the course of ischemic AKI, a variety of cell types (endothelial cells, neutrophils, T cells, macrophages, tubular cells, and pericytes) play important roles in injury and repair (4). However, injury to the proximal tubule cell (PTC) suffices to induce CKD and fibrosis (5), and injured tubular cells drive injury and inflammation by releasing profibrotic factors, in particular TGF $\beta$, and by producing inflammatory cytokines, including $\operatorname{TNF}_{\alpha}(6,7)$, identifying PTC as a major player in injury and fibrosis.

Tubular repair mechanisms involve the activation of EGF receptor (EGFR or HER1). While EGFR activation is beneficial in the early phases of epithelial repair in AKI (day 1-2 after injury), sustained activation of EGFR beyond the initial injury repair phase (days to weeks after injury) has been linked to kidney fibrosis in mouse models of bilateral ischemic AKI and unilateral ureteral obstruction (UUO) (813). Sustained activation of EGFR and its downstream pathways, in particular mitogen activated protein kinase (ERK), increases production of $\operatorname{TGF} \beta(9,13)$. TGF $\beta$ stimulates interstitial myofibroblasts (derived from stromal cells, most likely vascular pericytes; ref. 14) to proliferate and to secrete collagen and other matrix proteins, thus driving interstitial fibrosis and functional disruption of nephrons.

A disintegrin and metalloprotease 17 (ADAM17, also named TACE) releases the ectodomains of 
most EGFR ligands and of $\mathrm{TNF} \alpha$ from their transmembrane proforms to produce active soluble ligands (ectodomain shedding) (15). In vivo, in mouse and humans, pro-HER ligands and pro-TNF $\alpha$ represent ADAM17's physiologically dominant substrates (16, 17). Several EGFR ligands, including HB-EGF and EGF, have been associated with proliferative and migratory responses of tubular cells in vitro, and HB-EGF, TGF $\alpha$, and EGF have been shown to hasten tubular recovery and limit injury after AKI in vivo (18-20). Interestingly, HB-EGF and TGF $\alpha$, at the same time, have also been linked to glomerular sclerosis and kidney fibrosis (21-23), but it is unknown what causes these different outcomes. The role of other EGFR ligands, including epiregulin (EREG), epigen, amphiregulin (AREG), or betacellulin has not been examined in AKI or its transition to kidney fibrosis to date. The other main ADAM17 substrate, pro-TN$\mathrm{F} \alpha$, is an inflammatory cytokine and a major driver of kidney injury, promoting inflammation, apoptosis, and glomerular barrier damage (24). However, the effects of TNF $\alpha$ inhibition on kidney fibrosis and CKD progression are uncertain $(25,26)$. Thus, it is largely unknown which mechanisms sustain profibrotic activation of EGFR in the injured kidney, which specific HER ligands or other HER receptors are involved, and what role TNF $\alpha$ contributes. It is further unknown whether ADAM17 pathway upregulation is relevant in human AKI or CKD.

We report here that proximal tubule ADAM17 pathway upregulation and activation causes sustained EGFR activation and fibrosis after kidney injury (including ischemia and ureteral obstruction) via several cellular mechanisms in vitro and in vivo: ADAM17-induced upregulation of its own pathway (substrates and their receptors), unique profibrotic actions of its EGFR ligand substrate pro-AREG and EGFR-TNF $\alpha$ pathway crosstalk. Using 3 independent approaches/models targeting ADAM17, ADAM17 hypomorphic mice, specific ADADM17 inhibitor-treated WT mice, or mice with inducible genetic deletion of ADAM17 in the proximal tubule, we show strong protection against ADAM17 pathway upregulation and subsequent fibrosis. We further show that, in biopsy and urine samples of AKI and CKD patients with fibrosis, ADAM17 pathway activation also occurs and correlates significantly with markers of fibrosis, highlighting ADAM17 pathway inhibition as a potential therapeutic target in AKI and CKD in humans.

\section{Results}

ADAM17ex/ex mice show decreased fibrosis after bilateral ischemia reperfusion injury (IRI). ADAM17ex/ex hypomorphic mice with very low ADAM17 expression in all cells $(<5 \%$ of WT) have an introduced exon between exons 11 and 12 of the ADAM17 gene (17). This new exon not only carries an in-frame stop codon, but is also flanked by splice donor acceptor sites, which slightly deviate from the canonical sequences. Therefore, the new exon is used in over $95 \%$ of cases and leads to abortion of translation. Less than $5 \%$ of the time, full-length ADAM17 mRNA is produced; this keeps the animals viable. To examine the role of ADAM17 in AKI, we subjected WT and ADAM17ex/ex mice to severe bilateral IRI (clamping of both renal vascular supplies for 30 minutes) and sacrificed them at different time points after injury (day 1-42).

To assess the degree of initial injury, we compared upregulation of injury markers, tubular damage, and downregulation of CD31 staining as a marker of endothelial injury and cell loss in both genotypes. Serum creatinine (determined by mass spectometry) and blood urea nitrogen (BUN), 2 markers of glomerular filtration rate, were increased by IRI to similar values (creatinine: $1.02 \pm 0.12$ vs. $0.96 \pm 0.14 \mathrm{mg}$ / dl; BUN: $95.52 \pm 8.35$ vs. $87.74 \pm 5.82 \mathrm{mg} / \mathrm{dl}$ ) 24 hours after ischemia (Figure 1, A and B) and were, at no time during recovery, significantly different between the 2 groups. This recovery plateaued at about $3 \times$ baseline creatinine value $(0.2 \mathrm{mg} / \mathrm{dl})$ at $21-42$ days, indicating significant loss of kidney function (about $50 \%-70 \%$ glomerular filtration rate) as well as substantial and equal functional compensation in both genotypes (Figure 1, A and B). In both genotypes, urinary levels of kidney injury molecule 1 (KIM1) - a widely used kidney injury marker (Figure 1C) - and severe cortical tubular injury at 24 hours after ischemia (Figure 1D) were also similar. After 24 hours and up to 42 days after IRI, peritubular CD31-endothelial cell staining was decreased compared with sham-operated mice but did not show significant differences between ADAM17ex/ex and WT mice at any time tested, suggesting that peritubular capillary vessels and endothelial cells were equally preserved (Figure 1E). Collectively, these results indicate that the initial severe kidney injury was not significantly different between ADAM17ex/ex and their WT littermates.

Despite a similar degree of initial injury, other kidney outcomes varied significantly between WT and ADAM17ex/ex mice. On day 21 and 42 after injury, kidneys of ADAM17ex/ex mice showed strongly reduced production of the profibrotic markers $\alpha$ smooth muscle actin ( $\alpha \mathrm{SMA})$ and fibronectin, as detected by IHC (Figure 1F [day 21] and Supplemental Figure 1 [day 42]; supplemental material available online 


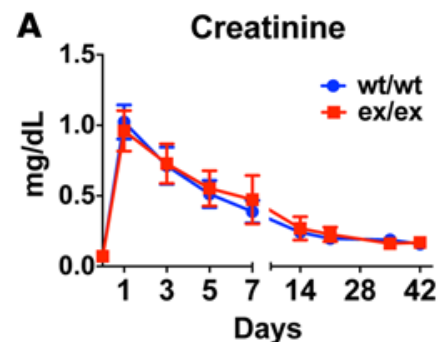

D

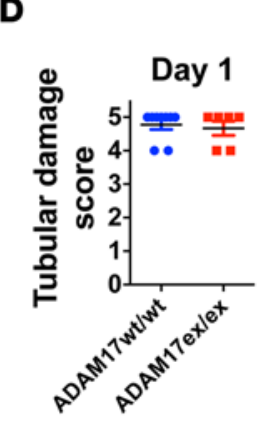

E
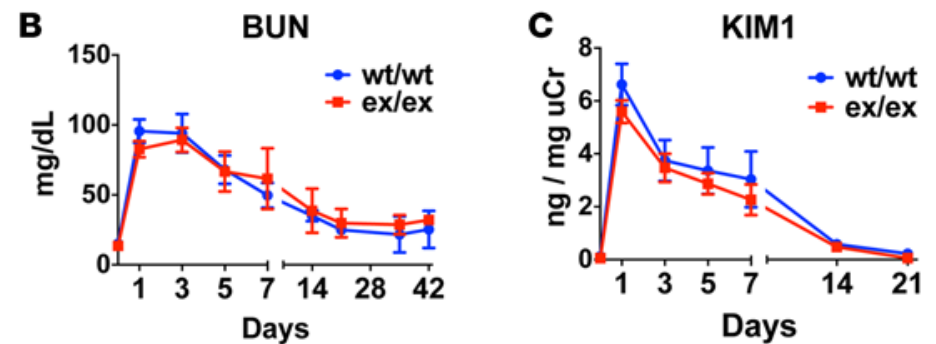

$\mathbf{F}$
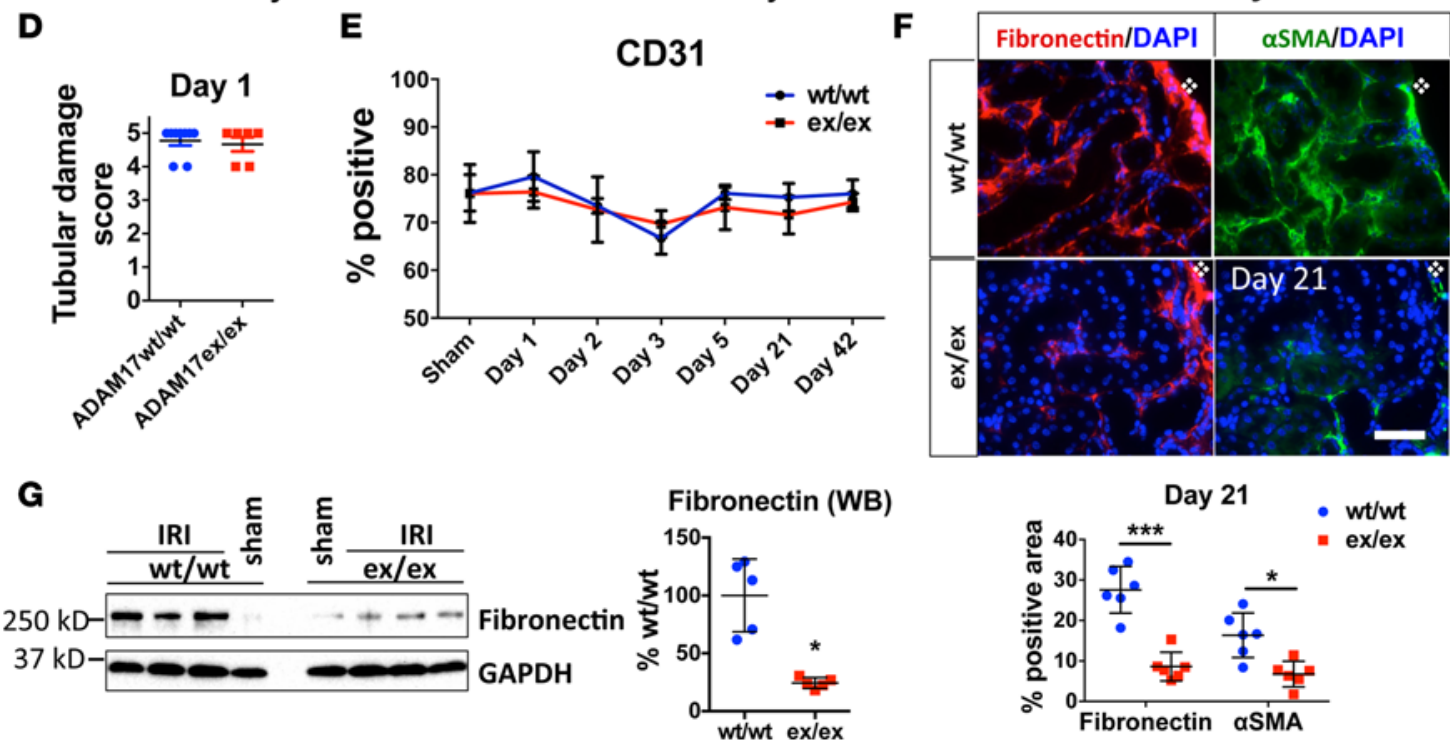

H

I
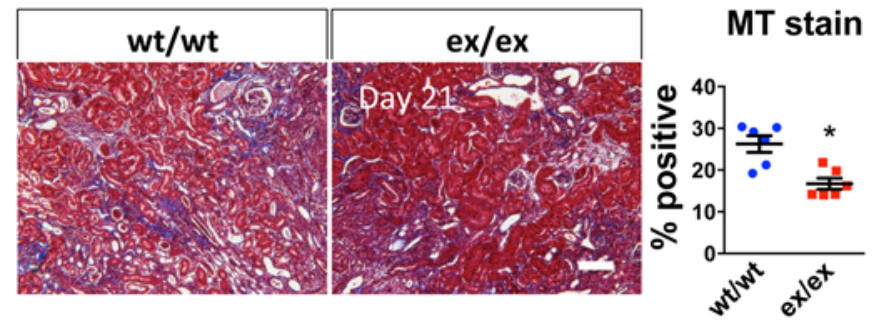

CD31
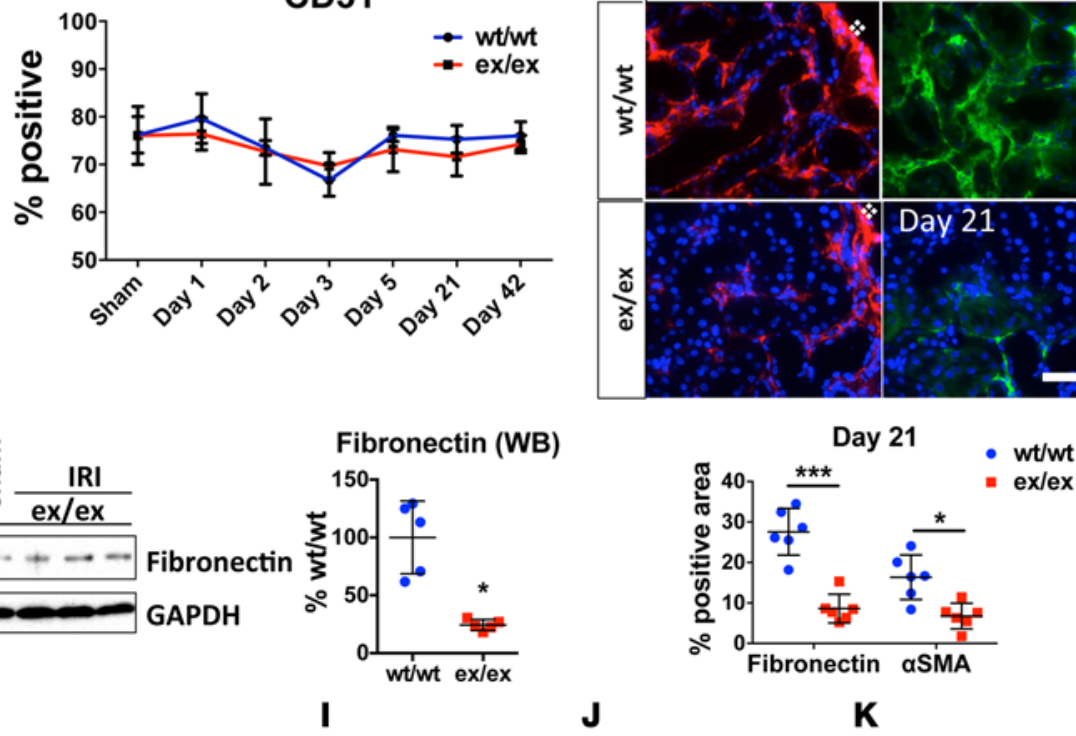

J
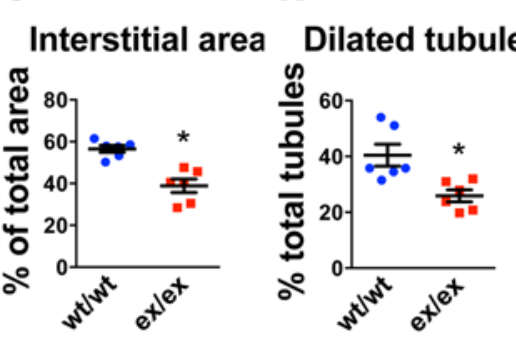

L
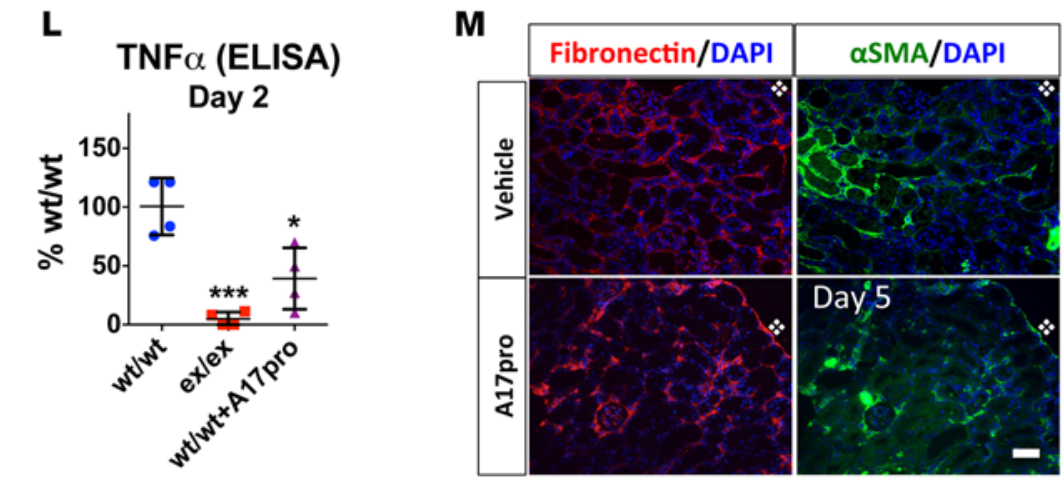

Figure 1. Global targeting of ADAM17 results in similar initial IRI-induced injury but in reduced IRI-induced fibrosis. ADAM17ex/ex mice and their WT littermates were subjected to bilateral ischemia for 30 minutes followed by reperfusion. (A and B) Time-course of changes in serum creatinine and BUN ( $n=6-15$ per time point). (C) Time-course of urinary KIM1 levels over time measured by ELISA ( $n=5-10$ per time point). (D) Tubular damage was scored in kidney cortex at day 1 after ischemia $(n=6-7)$. (E) Time course of cortical CD31 ${ }^{+}$area quantified after immunostaining $(n=4)$. (F) The induction of fibronectin and $\alpha$ SMA in kidney cortex was examined by immunostaining 21 days after injury (top: representative images; bottom: quantification, $n=6$; scale bar: $50 \mu \mathrm{m}$ ). (G) Fibronectin protein expression at day 21 after injury was examined by Western blot (left: sample blots; right: quantification; GAPDH was used as loading control; $n=5$ ). (H and I) Masson's trichrome staining at day 21 after injury in kidney cortex (H: representative images; I: quantification; $n=6$; scale bar: $100 \mu \mathrm{m})$. (J) Total interstitial area $(n=6)$ and (K) dilated tubules were quantified at day 21 after injury $(n=6)$. (L) Soluble TNF $\alpha$ was measured by ELISA in control (WT/WT), hypomorphic (ex/ex), and WT mice injected with recombinant ADAM17 
prodomain (WT/WT+A17pro) 2 days after injury $(n=4)$. (M) The induction of fibronectin and $\alpha$ SMA was examined in kidney cortex by immunostaining in vehicle or ADAM17 prodomain inhibitor-injected WT mice 5 days after injury (left: representative images, right: quantification; $n=4$; scale bar: 50 $\mu \mathrm{m}) .{ }^{*} P<0.05 ;{ }^{*} P<0.01 ;{ }^{* *} P<0.001$ as determined by an unpaired 2-tailed Student's $t$ test. Diamond symbol denotes position of kidney capsule. ADAM17, a disintegrin and metalloprotease 17; BUN, blood urea nitrogen; IRI, ischemia reperfusion injury; KIM1, kidney injury molecule 1; MT, Masson's trichrome; $\alpha \mathrm{SMA}$, alpha smooth muscle actin; TNF $\alpha$, tumor necrosis factor alpha; WB, Western blot.

with this article; doi:10.1172/jci.insight.87023DS1) and Western blot (Figure 1G). Consistent with this, ADAM17ex/ex mice showed reduced interstitial collagen deposition in the kidney cortex (Figure 1, $\mathrm{H}$ and I, Masson's stain), reduced overall expansion of interstitial area (Figure 1J), and a reduced number of dilated tubules (Figure 1K) - all characteristics of persistent tubulo-interstitial injury and disruption of structural nephron integrity. Thus, ADAM17 is involved in the development of delayed fibrosis after AKI injury, and the ADAM17 low state is protective.

ADAM17 prodomain inhibitor blocks ADAM17 substrate cleavage in vivo and confers protection against IRI-induced fibrosis. To ensure that the above described effects in ADAM17ex/ex mice depended on reduced ADAM17 catalytic activity, we treated WT mice with recombinant ADAM17 prodomain. The prodomain of ADAM17 blocks its catalytic site and is usually cleaved during trafficking of the molecule to the cell surface by a furin-type protease. It, however, can stay associated with the molecule on the cell surface until it is removed by a second cleavage event within the prodomain sequence carried out by an unknown protease (27). The prodomain used in our experiments has a mutated internal furin cleavage site and cannot be actively removed from the protease (28). When injected into tail vein or i.p., the prodomain inhibitor can be readily detected at high levels in various organs, including the kidney (data not shown). Compared with vehicle controls, daily i.p. prodomain injections resulted in reduced amounts of soluble TNF $\alpha$ ectodomain in kidney lysates (Figure 1L) and in reduced levels of $\alpha \mathrm{SMA}$ and fibronectin protein 5 days after ischemia (Figure 1M). These results confirmed that ADAM17 activity is required for its profibrotic function, but it remains unresolved as to which cells ADAM17 activity is most important in.

Proximal tubule-specific ADAM17 KO confers protection against fibrosis after IRI. Although EGFR deletion in most tubular cells (using a $\gamma$-glutamyltransferase 1-Cre $[\gamma$ GT-Cre] to confer relative specificity to the proximal tubule) confers protection against fibrosis in several kidney injury models $(9,22)$, the source of EGFR ligands and the specific tubular cell compartment of EGFR action remain unknown. Since the proximal tubule generates important kidney injury signals $(6,7)$ and ADAM17 is a ubiquitiously expressed protease, we first dissected its role in PTC.

We generated ADAM17 PTC-KO mice by crossing ADAM17 $7^{\mathrm{fl} / \mathrm{l}}$ mice with mice expressing Cre recombinase under control of the tamoxifen-inducible Slc34a1 promoter, a transport protein expressed only in the proximal S1/S2 segments of the PTC (29). We compared SLC34a1 $1^{\mathrm{GCE} /+}$ ADAM17 $7^{\mathrm{f} / \mathrm{fl}}$ (ADAM17 PTC-KO) with SLC34a1 ${ }^{\mathrm{GCE} /+}$ ADAM17 ${ }^{\mathrm{WT} / \mathrm{WT}}$ mice; Cre was present in both experimental groups, and all mice were injected with tamoxifen. In WT kidneys, ADAM17 was broadly expressed in proximal (PTC brush-border marker Lotus tetragonolobus lectin-positive [LTL-positive]; thick arrows) and distal tubule (LTL-negative; thin arrows) segments, as well as glomeruli and interstitial cells (Figure 2A; left panel). After tamoxifen injection into ADAM17 PTC-KO mice, ADAM17 was specifically deleted only in LTL-positive proximal tubule cells (thick arrows) but not in distal tubular segments (thin arrows) (Figure 2A, right panel). We then subjected ADAM17 PTC-KO mice and their WT littermates to bilateral IRI. Since the S3 segment is the most vulnerable PTC segment to ischemic injury, we ensured that our severe bilateral IRI protocol resulted in damage to all proximal tubule segments (S1-3) throughout the cortex, identical to our approach in ADAM17ex/ex mice.

Bilateral renal IRI caused similar injury and recovery as measured by injury markers BUN, NGAL, and KIM1 in both genotypes over time (Figure 2, B-D). However, as in our experiments with ADAM17 hypomorphic mice, functional recovery was only partial, and propensity to develop fibrosis differed substantially. Three weeks after ischemia, ADAM17 PTC-KO — as compared with WT littermates — showed significantly reduced fibronectin and $\alpha \mathrm{SMA}$ protein production (Figure 2E; immunofluorescence [IF]), reduced fibronectin protein expression (Figure $2 \mathrm{~F}$, Western blot), reduced interstitial collagen production (Figure 2, G and H, Masson's stain), less total interstitial area (Figure 2I), and reduced tubular dilation (Figure $2 \mathrm{~J}$ ). Thus, inducible specific ADAM17 KO in the very proximal segments of the proximal tubule can phenocopy, in terms of fibrosis, hypomorphic mice with very low ADAM17 in all cells, defining ADAM17 and its substrates localized in PTC as central players in IRI-induced kidney injury and fibrosis. 
A

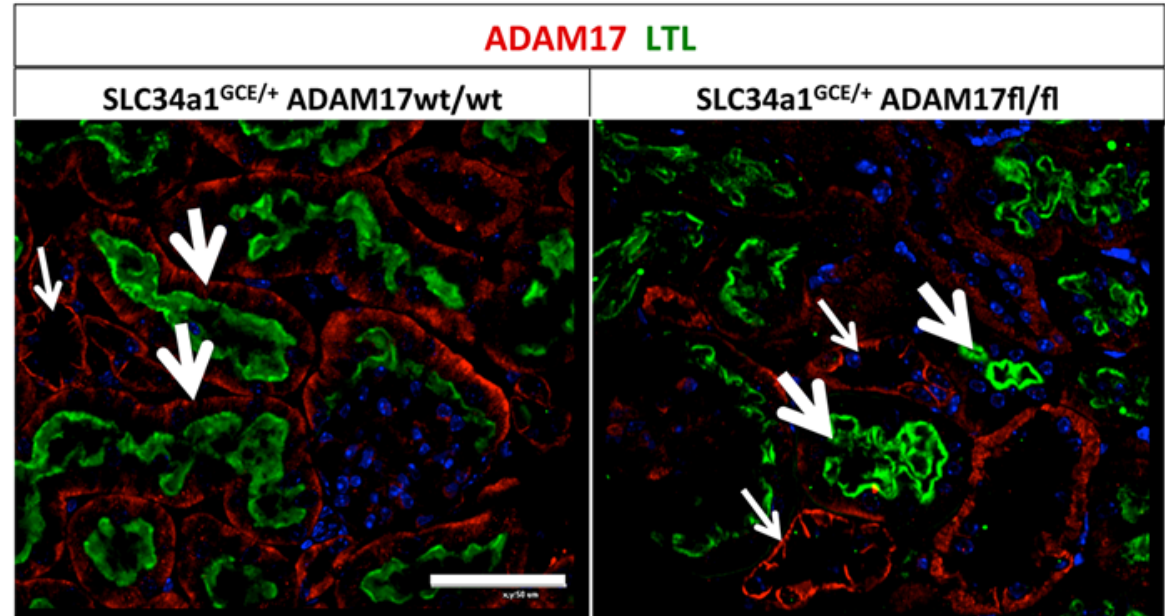

B

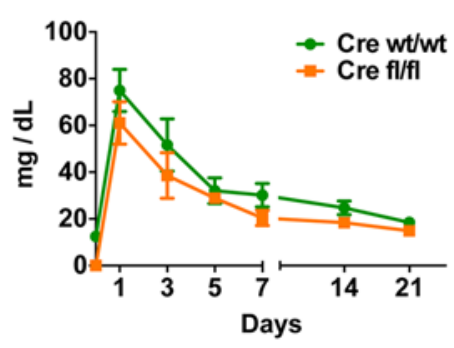

C

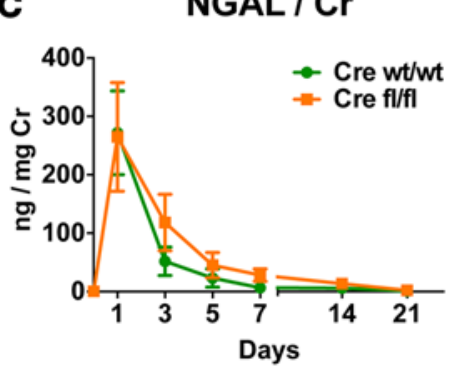

$\mathrm{KIM} 1 / \mathrm{Cr}$

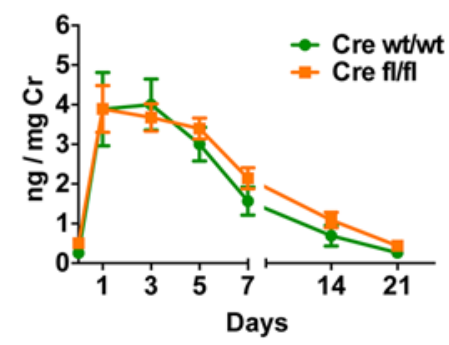

E

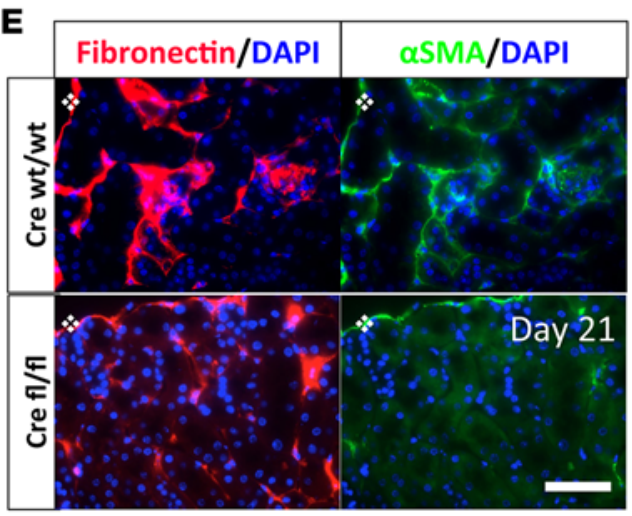

$\mathbf{F}$

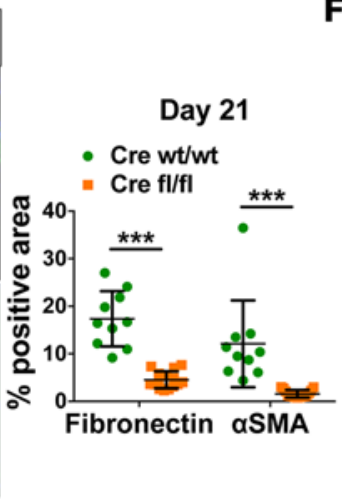

H

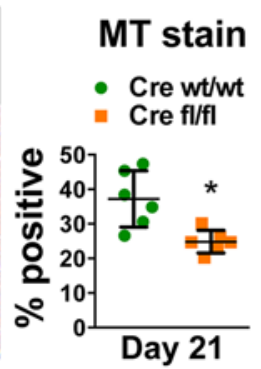

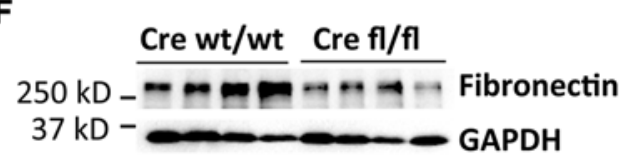

Fibronectin WB

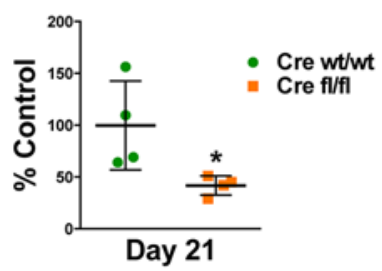

I J

\section{Interstitial area Dilated tubules}

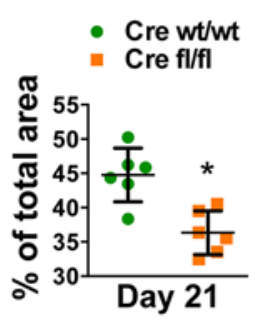

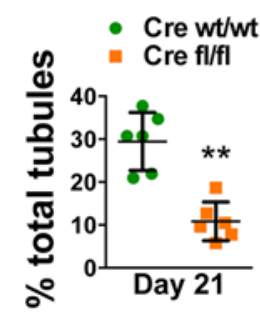

Figure 2. Proximal tubular-specific ADAM17 KO confers protection against fibrosis after IRI. Control (SLC34a $1^{\text {CCE } /+}$ ADAM17WT/WT, Cre WT/WT) and ADAM17 PTC-KO (SLC34a1 $1^{\mathrm{CCE} /+}$ ADAM17 ${ }^{\mathrm{fl} / \mathrm{fl}}$, Cre fl/fl) mice were subjected to bilateral ischemia for 29 minutes followed by reperfusion. (A) KO specificity was examined in healthy kidneys (left: control, right: PTC-KO mouse) by immunostaining of ADAM17 (red color) together with LTL staining (green color) as a proximal tubular marker (thick arrows, LTL-positive tubules; thin arrows, LTL-negative tubules; scale bar: $50 \mu \mathrm{m}$ ). (B-D) Time-course of BUN, urinary NGAL, and urinary KIM1 $(n=6-15)$. (E) The induction of fibronectin and $\alpha$ SMA was examined in kidney cortex by immunostaining at day 21 (left: representative images, right: quantification, $n=10$; scale bar: $50 \mu \mathrm{m}$ ). (F) Fibronectin protein expression at day 21 after injury was examined by Western blot (top: sample blots; bottom: quantification; GAPDH was used as loading control; $n=4)$. ( $\mathbf{G}$ and $\mathbf{H}$ ) Masson's trichrome staining in kidney cortex at day 21 after injury (G: 
representative images; $\mathbf{H}$ : quantification; $n=6$; scale bar: $100 \mu \mathrm{m})$. (I) Total interstitial area $(n=6)$ and $(\mathbf{J})$ dilated tubules $(n=6)$ were quantified in kidney cortex at day 21 after injury. ${ }^{*} P<0.05 ;{ }^{* *} P<0.01$; ${ }^{* *} P<0.001$ as determined by an unpaired 2-tailed Student's $t$ test. Diamond symbol denotes position of kidney capsule. ADAM17, a disintegrin and metalloprotease 17; BUN, blood urea nitrogen; IRI, ischemia reperfusion injury; KIM1, kidney injury molecule 1; LTL, Lotus Tetragonolobus lectin; MT, Masson's trichrome; NGAL, neutrophil gelatinase-associated lipocalin; PTC-KO, proximal tubule cell KO; $\alpha$ SMA, alpha smooth muscle actin; WB, Western blot.

ADAM17ex/ex and PTC-KO mice are protected against fibrosis caused by ureteral obstruction. To test whether protection against fibrosis extended to other injuries beyond ischemia, we used a different model of kidney fibrosis, UUO. ADAM17ex/ex mice and WT littermates were subjected to UUO and examined after 7 and 14 days. Similar to IRI, ADAM17ex/ex mice injured with UUO showed reduced $\alpha$ SMA and fibronectin at the mRNA (Figure 3A) and protein levels (Figure 3B, IF, and Figure 3C, Western blot) and significantly reduced interstitial fibrosis as compared with WT mice (Figure 3D; Masson's stain). Very similar results were obtained in ADAM17 PTC-KO mice subjected to UUO (Figure 3, E-H), suggesting that the ADAM17 pathway in PTC represents a central signaling hub relevant to kidney fibrosis that responds to various tubular injuries.

Genetic deletion of ADAM17 in kidney stroma (stroma-KO) using FoxD1-Cre (Supplemental Figure 2A; KO specificity verified by immunostaining; ref. 14) conferred no substantial protective effect against fibrosis induced by UUO (Supplemental Figure 2B), suggesting that myofibroblasts are not a major source of ADAM17-cleaved signals that promote fibrosis in this injury model.

Injury-induced ADAM17 pathway upregulation causes sustained EGFR activation in WT mice, while this effect of injury is impaired in ADAM17ex/ex mice. In order to identify which ADAM17 substrates and which of their receptors carry out the observed ADAM17 profibrotic function, we examined its pathway induction in vivo. In WT mice, kidney injury (IRI or UUO) caused upregulation of the entire ADAM17 pathway, including ADAM17, its EGF ligand, and $\mathrm{TNF} \alpha$ substrates, as well as their receptors.

In WT mice, ADAM17 whole kidney mRNA expression was strongly increased 2 days after injury (more than 5-fold compared with sham-operated mice), remained elevated for at least 21 days, and returned close to baseline by 42 days (Figure 4A). However, in ADAM17ex/ex mice, no ADAM17 mRNA increase was detectable for at least 5 days after ischemia. Only by 21 days, we detected a moderate increase (about 2.7-fold over sham), but ADAM17 mRNA returned to almost baseline at 42 days (Figure 4A). Similarly, after UUO, ADAM17 mRNA levels were elevated at day 7 in WT mice and remained low in ADAM17ex/ ex mice (Supplemental Figure 3A). Consistent with the detected mRNA levels, ADAM17 protein was strongly increased by ischemia in WT mice (Figure 4B) and remained elevated for at least 21 days (data not shown), whereas it was hardly detectable in ADAM17ex/ex mice (Figure 4B) except for a moderate elevation at late time points at 21 days after injury (data not shown). ADAM17 upregulation at late time points occurs due to the fact that, in hypomorphic mice, a full-length ADAM17 protein is still produced less than $5 \%$ of the time and indicates that mechanisms of ADAM17 upregulation after injury are principally intact in ADAM17ex/ex mice.

The mRNAs of ADAM17 substrate pro-EGFR ligands (HB-EGF, AREG, EREG, TGF $\alpha$ ) and pro-TN$\mathrm{F} \alpha$, as well as their corresponding receptors EGFR and TNFR1/2, were significantly increased by injury in WT mice, but this effect was blunted in ex/ex mice. In WT mice, AREG was substantially more upregulated on day 1-21 than any other EGFR ligand in both mouse models tested (Figure 4C [IRI] and Supplemental Figure 3B [UUO]) while mRNA expression of the EGFR ligand and ADAM10 substrate EGF remained at very low levels after injury (Figure 4C [IRI] and Supplemental Figure 3B [UUO]). In contrast, ADAM17ex/ ex mice showed only very mild to moderate increases of EGFR ligand mRNA expression on day 1 after injury; the expression returned to almost basal levels by day 2 and remained low for at least 5 days after injury (Figure 4D [IRI] and Supplemental Figure 3B [UUO, day 7]). On the receptor level, EGFR was strongly upregulated in injured WT mice over at least 5 days, but not in ex/ex mice, which only showed a moderate increase by day 21 (Figure 4E [IRI] and Supplemental Figure 3A [UUO, day 7]). TNF $\alpha$ and its receptors followed a similar pattern. TNF $\alpha$ and TNFR1/2 in WT mice were strongly increased by injury on day 1-2 and remained high for at least 5 days; TNF $\alpha$ and TNFR1/2 expression in ex/ex mice was moderately elevated similar to WT mice on day 1 , returned to baseline levels by day 2 , and only showed a moderate late second peak of expression increase by day 5 (Figure 4, F-H [IRI], and Supplemental Figure 3A [UUO]).

As a result of these differences in transcriptional and translational factor upregulation (mRNA and protein level), ADAM17ex/ex mice as compared with WT mice also showed strong differences in post- 
A

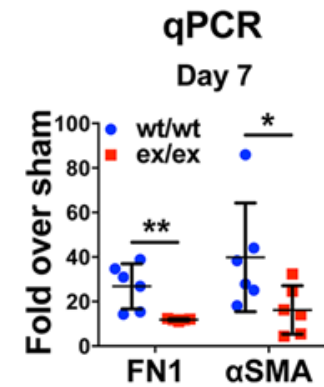

B

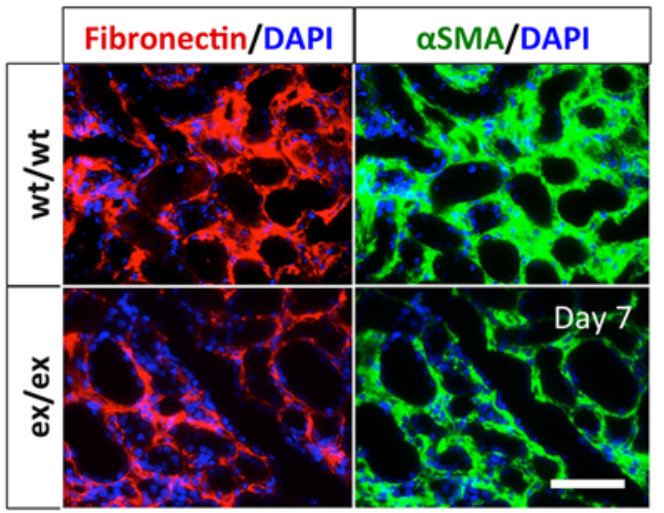

D
C

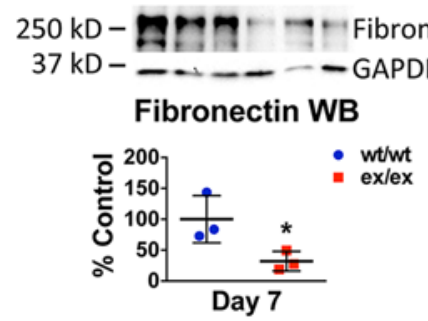

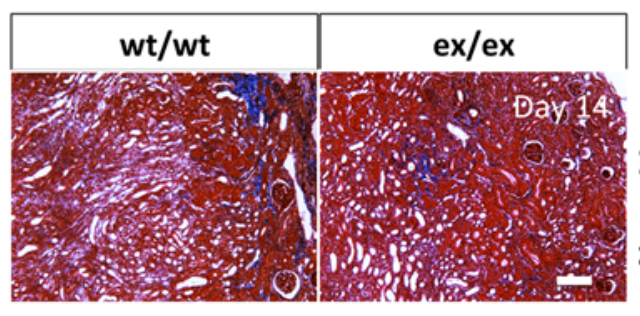

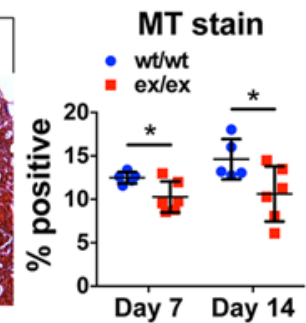

Day 7 Day 14
E

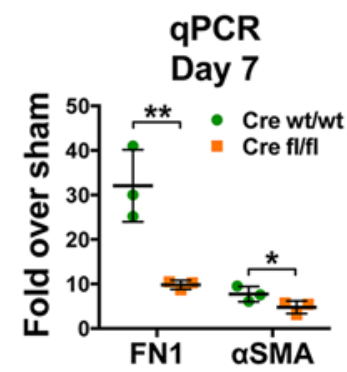

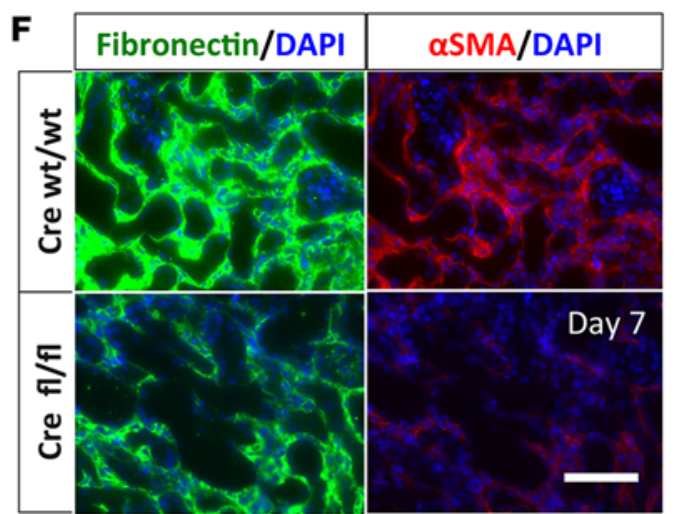

\section{Day 7}

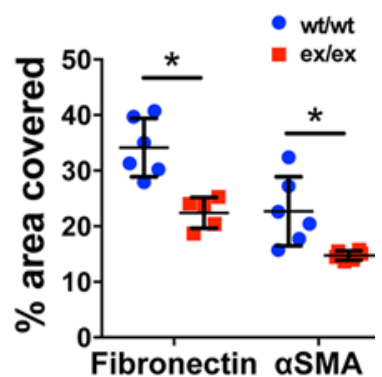

G

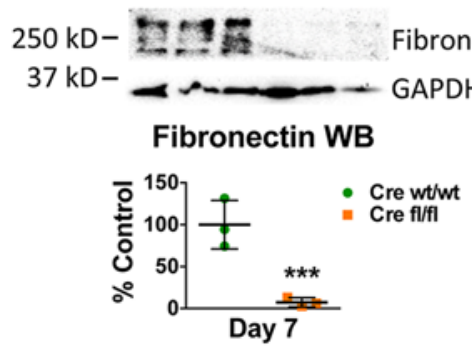

H

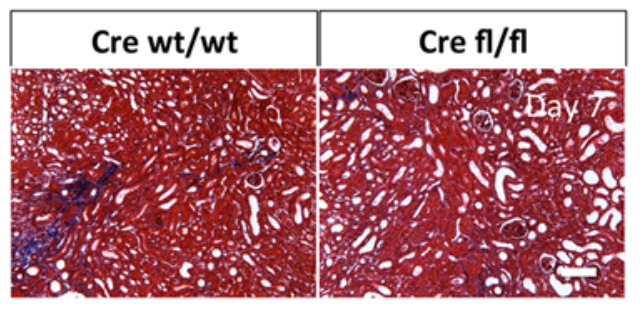

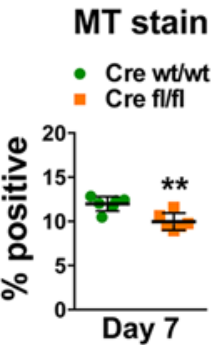

Figure 3. ADAM17ex/ex and ADAM17 PTC-KO mice are protected from UUO-induced fibrosis. Mice were subjected to UUO, and their kidneys were examined 7 or 14 days after ureteral ligation, as noted. (A) qPCR analysis of fibronectin or $\alpha$ SMA mRNA expression levels in whole kidneys of ex/ex or WT/WT mice at day 7, expressed as fold over respective sham-injured mice $(n=6)$. (B) The induction of fibronectin and $\alpha$ SMA in the injured kidney cortex of UUO-subjected WT/WT or ex/ex mice was examined by immunostaining (left: representative images; right: quantification; $n=6$; scale bar: $50 \mu \mathrm{m})$. (C) Fibronectin protein expression at day 7 after ligation was examined by Western blot (top: sample blots; bottom: quantification; GAPDH was used as loading control; $n=3$ ). (D) Masson's trichrome staining in kidney cortex at day 7 or day 14 after ligation (left: representative images day 14; right: quantification; $n=5-6$; scale bar: $100 \mu \mathrm{m}$ ). (E) qPCR analysis of fibronectin or aSMA mRNA expression levels in whole kidneys of UUO-subjected control (Cre WT/WT) or ADAM17 PTC-KO (Cre fl/fl) at day 7, expressed as fold over respective sham-injured mice $(n=3)$. (F) The induction of fibronectin and $\alpha$ SMA in the injured kidney cortex of UUO-subjected control (Cre WT/WT) or ADAM17 PTC-KO (Cre fl/fl) mice was examined at day 7 by immunostaining (left: representative images, right: quantification; $n=5$; scale bar: $50 \mu \mathrm{m}$ ). (G) Fibronectin protein expression at day 7 after ligation was examined by Western blot (top: sample blots; bottom: quantification; GAPDH was used as loading control; $n=3$ ). (H) Masson's trichrome 
staining in kidney cortex at day 7 after ligation in control (Cre WT/WT) or ADAM17 PTC-KO (Cre fl/fl) mice (left: representative images; right: quantification; $n=6$; scale bar: $100 \mu \mathrm{m}$ ). ${ }^{*} P<0.05$; ${ }^{* *} P<0.01 ;{ }^{* *} P<0.001$ as determined by an unpaired 2-tailed Student's $t$ test. ADAM17; a disintegrin and metalloprotease 17; FN1, fibronectin; MT, Masson's trichrome; PTC-KO, proximal tubule cell KO; $\alpha$ SMA, alpha smooth muscle actin; UUO, unilateral ureteral obstruction; WB, Western blot.

translational modification of ADAM17 substrates, with much reduced TNF $\alpha$ and EGFR ligand cleavage in vivo after injury (Figure 4I; ADAM10 substrate cMet is shown as a control) and strongly reduced or absent phosphorylation of EGFR on days 1-5 after injury, examined by Western blot and immunostaining (Figures 4, J and K [IRI], and Supplemental Figure 3C [UUO]). Further, using the ADAM17 PTC-KO mouse model, we also saw very similar upregulation of the ADAM17 pathway in WT littermates subjected to both IRI or UUO, with AREG being the most upregulated EGFR ligand ( 100 -fold higher than any other EGFR ligand; data not shown).

In conclusion, the absence of injury-induced ADAM17 upregulation and lack of its cleavage activity appears to reduce upregulation of its downstream signaling pathways, suggesting the existence of positive-feedback loops. We hypothesized based on our observations in vivo that ADAM17 and its substrates produce ligands that, in turn, enhance their own transcriptional upregulation or activity and involve, in particular, the action of AREG (EGFR ligand) as well as the TNF $\alpha$ pathway, leading to sustained profibrotic EGFR activation.

AREG more than any other EGFR ligand causes ADAM17 pathway upregulation and expression of profibrotic and proinflammatory mediators in vitro in mouse and human PTC; crosstalk with the TNFa pathway strongly potentiates this effect. For the in vitro analysis of ADAM17 dependent pathways, we used mouse primary tubular cells isolated from WT and ADAM17ex/ex mice, as well as a human PTC line (HPTC). AREG, in comparison to any other EGFR ligand, and also TNF $\alpha$, potentiated the expression of their own pathway components (the effect of all ADAM17 EGF ligand substrates tested is shown in Supplemental Figure 4). Thus, AREG induced the expression of AREG itself, ADAM17, TGF $\alpha$, and HB-EGF, while TNF $\alpha$ induced the expression of TNF $\alpha$ itself and TNFR2 (Figure 5A). More importantly, however, we found significant crosstalk between both pathways, likely potentiating their individual injurious actions in vitro and in vivo.

Exposure of HPTC to TNF $\alpha$ resulted in significant upregulation of mRNA expression of EREG, as well as of AREG (Figure 5A). Conversely, AREG potently induced the expression of TNFR2 (Figure 5A). These results confirm the hypothesis that ADAM17-cleaved ligands, in particular AREG and TNFa, drive positive-feedback loops that enhance expression and activation of their own individual pathways; however, both ADAM17-dependent pathways also crosstalk and synergize in their actions. This synergistic action can, for example, be observed on the level of the production of profibrotic and proinflammatory cytokines. When compared with any other EGFR ligand (added in isomolecular amounts), AREG stimulated significantly more mRNA production of TGF $\beta$, a potent profibrotic cytokine, as well as of monocyte chemoattractant protein 1 (MCP1), of macrophage inflammatory protein 1 alpha (MIP1A), and of regulated on activation normal $t$ cells expressed and secreted (RANTES) - cytokines involved in monocyte/macrophage recruitment after kidney injury (Figure 5B and Supplemental Figure 4). TNF $\alpha$, as expected, also strongly induced the same proinflammatory molecules; however, when used in combination with AREG, its effects were significantly potentiated (Figure 5B). Interestingly, TNF $\alpha$ stimulation caused AREG cleavage in HPTC (Figure 5C), suggesting an important upstream crosstalk mechanism that would potentiate and sustain EGFR activation and its profibrotic action. These results strongly suggest that the profibrotic function of ADAM17 is due to the concerted injurious actions of its EGFR ligand substrates, in particular AREG, and of TNF $\alpha$.

AREG causes sustained EGFR activation in proximal tubule cells in a cleavage-dependent manner. Whether different ligands have differential effects on activation of EGFR and its downstream pathways, and which are the implicated mechanisms, is not well understood. We exposed HPTC to isomolecular amounts of AREG and HB-EGF, the ligands with the strongest and the weakest effect on EGFR pathway induction in HPTC (Supplemental Figure 4), and examined their effects on EGFR activation, as well as the activation of EGFR downstream targets. HB-EGF induced fast, strong, short-lasting phosphorylation of EGFR at Y1068 starting at 5 minutes and returning to baseline levels by 4 hours (Figure 5, D and G). In contrast, AREG resulted in a biphasic phosphorylation pattern of EGFR and its downstream effectors ERK1/2, with mild-to-moderate early phosphorylation and a second peak of long-lasting phosphorylation starting at about 8 hours and lasting at least 24 hours (Figure 5, E and G). To test whether the second peak of EGFR 
A

ADAM17

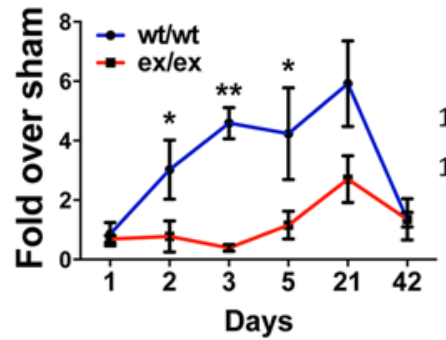

C

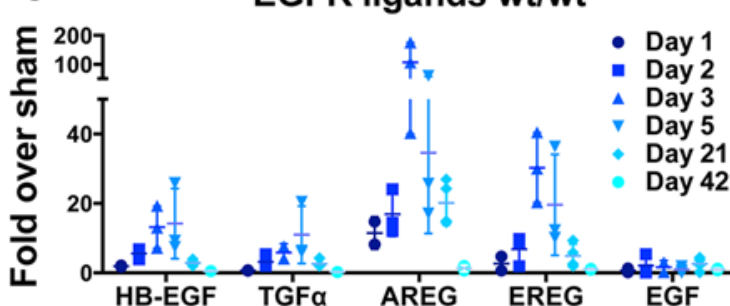

E

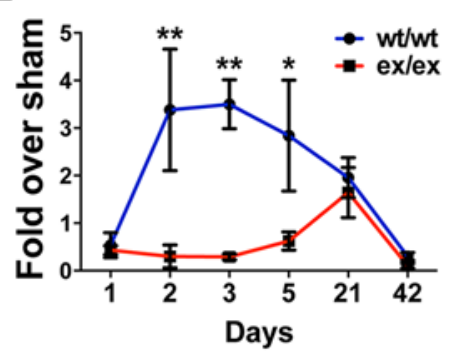

B $\frac{\text { sham }}{\sum_{\frac{x}{3}}^{*} \frac{\text { IRI }}{x} \frac{w t / w t}{\text { ex/ex }}}$

$-\quad \leftarrow$ pro-ADAM17

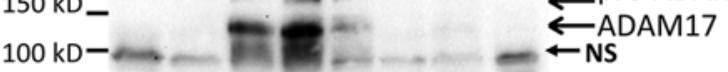

$37 \mathrm{kD}-$

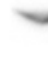

GAPDH
D
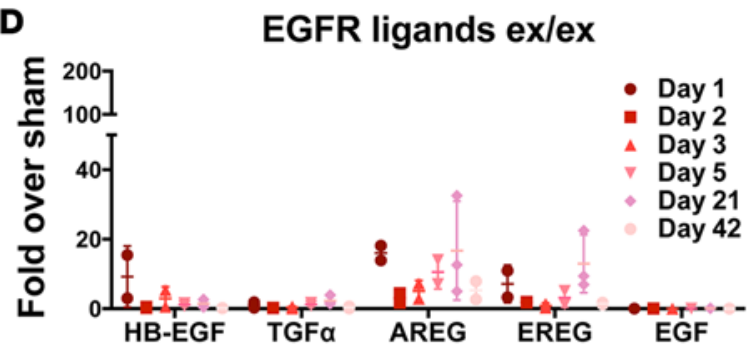

F

TNF $\alpha$

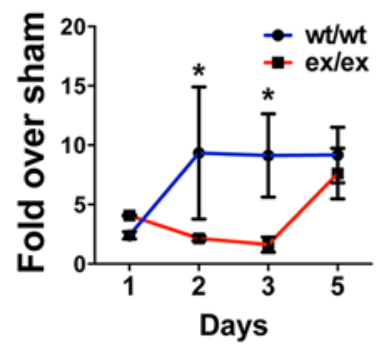

G

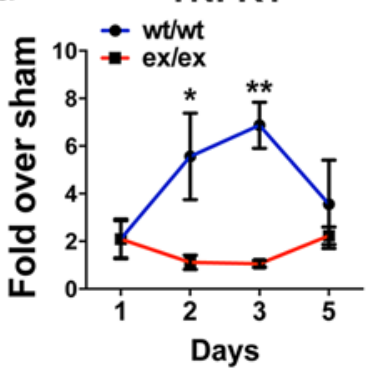

J

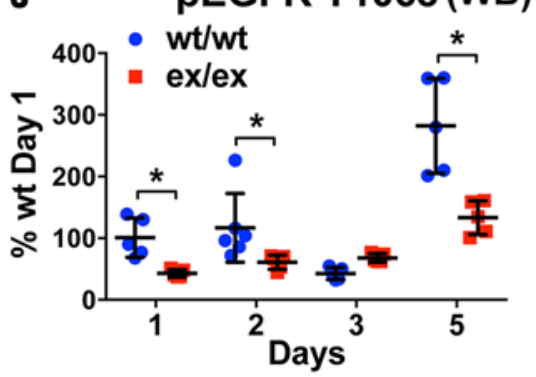

H

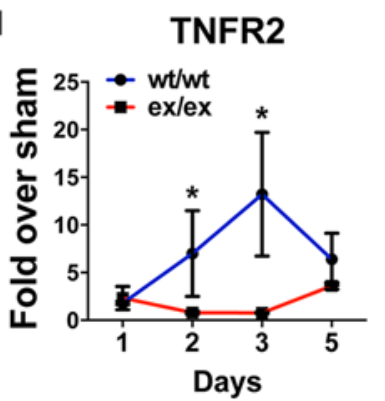

I
ADAM17 WB

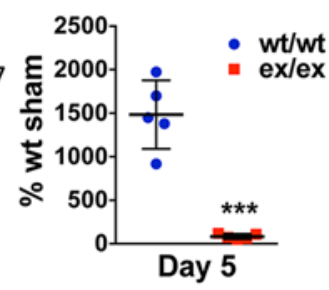


mice at day 2 after ischemia by ELISA (ADAM10 substrate cMET is used as control; $n=4$ ). (J) Time-course of EGFR phosphorylation (Y1068) as measured by Western blot in whole kidney lysates of WT/WT or ex/ex mice $(n=5)$. (K) Representative images of EGFR phosphorylation (Y1173) examined in kidney cortex by immunostaining in WT/WT or ex/ex mice at day 2 after injury (left: representative images; right: quantification; $n=3 ; \mathrm{scale}$ bar: $50 \mu \mathrm{m}) .{ }^{*} P<$ $0.05 ;{ }^{* *} P<0.01 ;{ }^{* *} P<0.001$ as determined by an unpaired 2-tailed Student's $t$ test. ADAM17; a disintegrin and metalloprotease 17; EGFR, epidermal growth factor receptor; IRI, ischemia reperfusion injury; NS, nonspecific band; TNF $\alpha$, tumor necrosis factor $\alpha$; TNFR, tumor necrosis factor receptor; AREG, amphiregulin; ADAM10, a disintegrin and metalloprotease 10; cMet, met proto-oncogene; WB, Western blot.

phosphorylation requires the cleavage of EGFR ligands, we exposed HPTC to AREG in the presence of the metalloprotease inhibitor BB94. We found that, although the initial EGFR phosphorylation peak is not affected (as expected), the second peak is abolished (Figure 5, F and G). These results suggest that AREG sustains EGFR activation in PTC in vitro (and likely in vivo) via a positive feedback loop that includes upregulation of the expression (Figure 5A) and subsequent cleavage (Figure 5, C, F, and G) of EGFR ligands (as measured by EGFR phosphorylation), including prominently AREG itself.

Injury-induced cytokine expression, as well as kidney ingress of neutrophils and macrophages, is reduced in vivo in ADAM17ex/ex and ADAM17 PTC-KO mice. Given these observed effects of soluble ADAM17 substrates on the production of profibrotic and proinflammatory cytokines in PTC in vitro, we also examined the induction of these cytokines after injury in vivo. ADAM17ex/ex mice showed reduced levels of both TGF $\beta$ and MCP1 expression at 1-5 days after ischemia compared with WT littermates (Figure 6, A and B). Reduced production of TGF $\beta$ correlated with reduced levels of $\alpha$ SMA and fibrosis in all our injury models (Figures 1-3) and with the reduced induction of the myofibroblast marker PDGFR $\beta$ (Figure 6C). Reduced levels of proinflammatory cytokines were associated with significantly reduced accumulation of immune cells in the kidney. As compared with WT mice, macrophage ingress into the kidney — quantified by F4/80 staining - was diminished in ADAM17ex/ex mice at 3, 5, or 21 days after ischemia, as well as at 7 days after UUO (Figure 6D). Neutrophil infiltration at days 1 and 5 after ischemia was also reduced in ADAM17ex/ ex mice (Figure 6E). In ADAM17 PTC-KO mice, as compared with their WT littermates, we only saw a nonsignificant trend of reduction of macrophage ingress in IRI experiments at the time points measured, but we detected a significant reduction at day 7 in the UUO experiments (Figure 6F). In terms of neutrophil ingress, we detected a significant reduction at day 1 after IRI when neutrophil levels were high in WT mice, while no differences between WT mice and ADAM17 PTC-KO mice were detectable at day 5, when both genotypes showed very low levels of neutrophils (Figure 6G).

In conclusion, injury-induced proximal tubule ADAM17 substrate cleavage in vivo - presumably predominantly involving AREG and TNF $\alpha$ as shown in our in vivo and in vitro experiments - leads to the release of proinflammatory cytokines, resulting in recruitment of neutrophils and macrophages and the production of the profibrotic cytokine TGF $\beta$, which together drive fibrosis.

ADAM17, AREG, and TNFR1 expression and EGFR phosphorylation are increased in human AKI and $C K D$. To examine whether ADAM17-dependent pathways, as suggested by our mouse studies, are also induced in human kidney disease, we examined the expression levels of ADAM17 and AREG and the activation of EGFR in human kidney biopsies (Brigham and Women's Hospital kidney biobank). We tested 3 different diagnosis - 4 patients each — and show representative images in Figure 7: hyperoxaluria (HOX; intraluminal tubular obstruction of urine flow as it occurs in crystal-forming oxaluria patients represents a more proximal but pathogenetically similar mechanism to UUO in mice), glomerulonephritis (GN), and diabetic nephropathy (DN). Most samples showed evidence of tubulo-interstitial fibrosis as determined by a pathologist (based on Masson's trichrome stain) and also by immunostaining for the fibrotic marker $\alpha \mathrm{SMA}$ as compared with control samples (Figure 7A, first column). Similar to mice, we found that tubular and interstitial ADAM17 expression, tested by immunostaining, was strongly increased in most human kidney tissue samples (Figure 7A, second column). Concurrently, we found increased expression of AREG (Figure 7A, third column), as well as increased activation of EGFR, as measured by a phosphospecific EGFR antibody (Figure 7A, fourth column). Interestingly, the amount of the profibrotic marker $\alpha$ SMA expressed in tissue samples across all patients and conditions was positively and strongly correlated with ADAM17 or AREG expression (Figure 7, B and C), suggesting a strong link between ADAM17 expression level and fibrosis in human kidney disease.

In addition, we examined the levels of ADAM17-cleaved substrates in urine sample of AKI and CKD patients (Figure 8; see also patient characteristics in Supplemental Table 1). Interestingly, soluble AREG and TNFR1 were very significantly and strongly upregulated in the urine samples of patients 
A

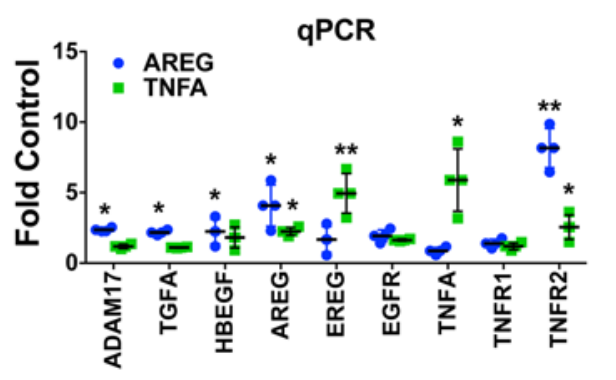

B
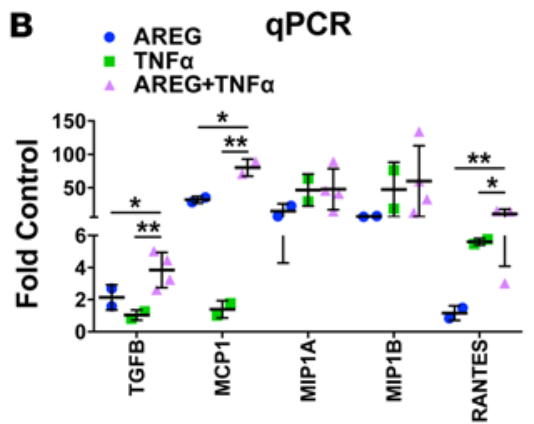

C
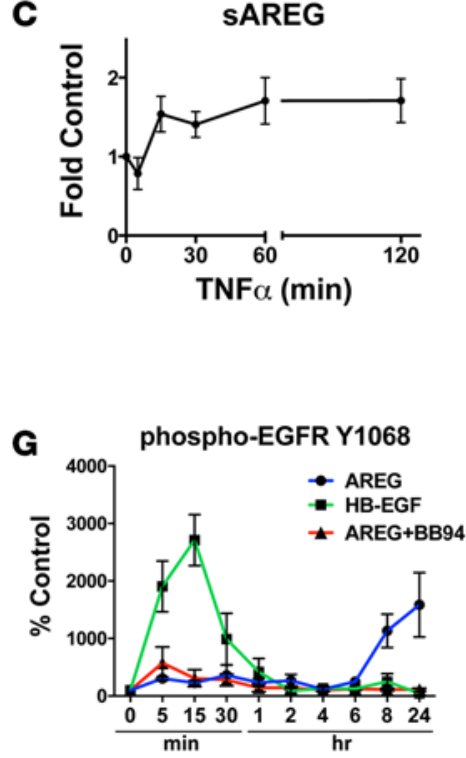

D

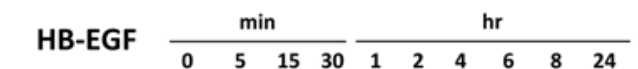

$\mathbf{E}$

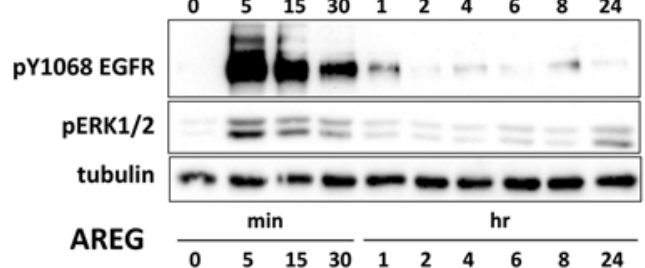

pY1068 EGFR , ,

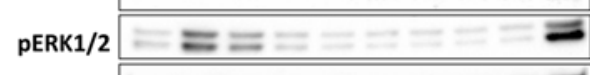

tubulin

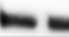

$\mathbf{F}$

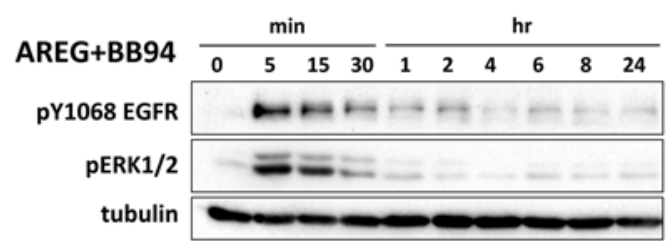

Figure 5. Amphiregulin (AREC) and TNFa crosstalk causes sustained EGFR activation and strongly enhanced cytokine production in human proximal tubular cells. ( $A$ and $B$ ) qPCR analysis of ADAM17 pathway components (A) and proinflammatory/profibrotic cytokines (B) in human proximal tubular cells treated with AREG, TNF $\alpha$, or both (AREG+T$N F \alpha)$ for 24 hours $(n=4)$. (C) Time course of TNF $\alpha$-induced AREG cleavage producing soluble AREG (sAREG) measured by ELISA in HPTC culture medium $(n=3)$. (D-F) Time course of HB-EGF-induced (D) or AREG-induced (E and F) Y1068-EGFR and ERK1/2 phosphorylation (analyzed by Western blot) in the absence ( $\mathbf{D}$ and $\mathbf{E}$ ) or presence $(\mathbf{F})$ of the metalloprotease inhibitor BB94. Tubulin is used as loading control for quantification (G; $n=3) .{ }^{*} P<0.05 ;{ }^{* *} P<0.01$ as determined by an unpaired 2-tailed Student's $t$ test. ADAM17, a disintegrin and metalloprotease 17; AREG, amphiregulin; BB94, batimastat; EGF, epidermal growth factor; EGFR, epidermal growth factor receptor; EREG, epiregulin; ERK, extracellular regulated mitogen activated protein kinase; HB-EGF, heparin binding epidermal growth factor-like growth factor; HPTC, human proximal tubule cells; MCP1, monocyte chemoattractant protein 1; MIP1A, macrophage inflammatory protein 1 alpha; MIP1B, macrophage inflammatory protein 1 beta; RANTES, regulated on activation normal T cell expressed and secreted; TNF $\alpha$, tumor necrosis factor alpha; TNFR, tumor necrosis factor receptor.

with severe AKI and tubular necrosis (Figure 8, A and B), a condition prone to the development of fibrosis, in mice (as shown in our experiments) and in humans (4, 30, 31). AREG and TNFR1 were also moderately upregulated in the urine of patients with fibrotic CKD (many of them diabetics) (Figure 8, A and B). AREG, in fact, was the only EGFR ligand tested that was significantly upregulated under both of these conditions. HB-EGF was modestly but significantly upregulated in the AKI group (Figure 8C), and TGF $\alpha$ was upregulated in none of the conditions as compared with control (Figure 8D). We failed to detect the presence of soluble TNF $\alpha$ in human urine samples, but the presence of soluble TNFR1 suggests activity of ADAM17 and the TNF $\alpha$ pathway in human AKI and CKD, since - besides TNF $\alpha$ - its receptors TNFR1 and TNFR2 are also ADAM17 substrates $(32,33)$. In accordance with our results in mice, these findings thus suggest a possible involvement of ADAM17 and activation of its main physiological downstream pathways, EGFR (likely via AREG) and TNF $\alpha$ /TNFR, in AKI and chronic fibrotic kidney disease in humans. A summary of our results is provided in a schematic in Figure 9.

\section{Discussion}

Our results demonstrate the protective effect of inhibition of ADAM17-mediated release of EGFR ligands and TNF $\alpha$ against kidney fibrosis that develops after different kidney injuries (AKI, UUO), suggesting a central role of ADAM17 in fibrotic kidney diseases. Using an inducible ADAM17 KO specific to only the first 2 segments of the proximal tubule (Slc34a1-Cre), we identified the proximal tubule as the source of EGF ligands that drives tubular EGFR activation in fibrosis. Our results corroborate other reports that show that sustained tubular EGFR activation after AKI or UUO drives kidney fibrosis in WT mice, while mice with low EGFR activity (wa2 mice), EGFR inhibition, or EGFR deletion in tubular cells ( $\gamma$ GT-Cre) were protected $(9,11,13)$.

Some studies reported that AKI-induced fibrosis is mediated by ligand-independent activation of EGFR by SRC kinase at Y845 and might involve production of ROS $(9,34)$. Our results, however, sug- 

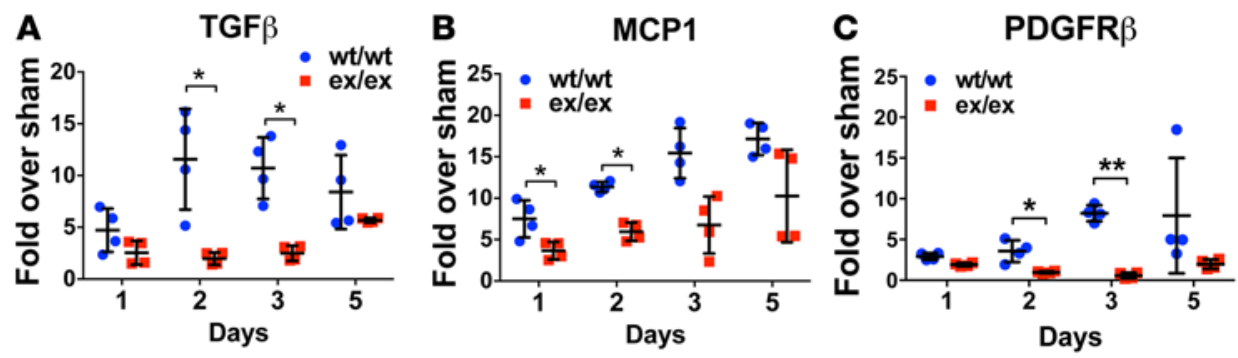

D

Macrophage (F4/80)

IRI

UUO
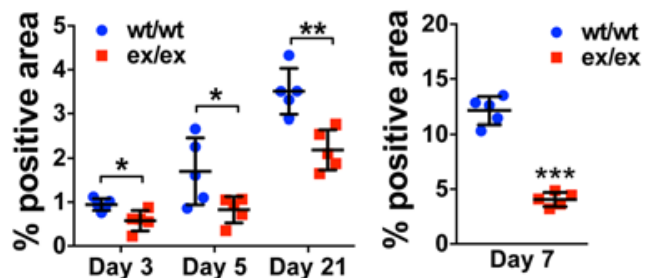

F

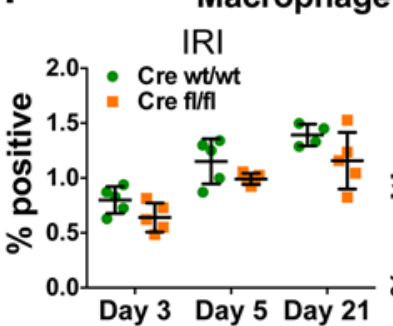

Macrophage (F4/80)

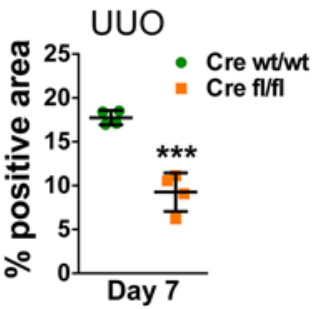

\section{E Neutrophil (Ly6G)}

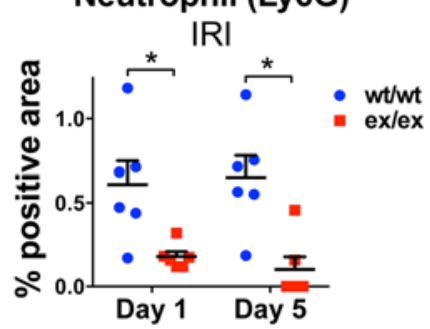

G Neutrophil (Ly6G)

IRI

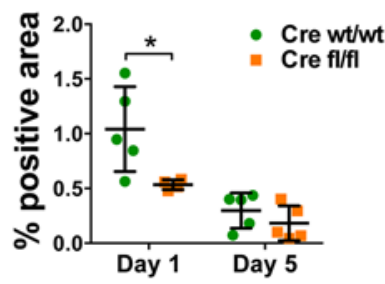

Figure 6. Injury-induced cytokine expression and kidney ingress of macrophages and neutrophils is reduced in vivo in ADAM17ex/ex and ADAM17 PTC-KO mice. (A-C) The mRNA expression of TCF $\beta$, MCP1, and PDCFR $\beta$ was examined by qPCR in whole kidney lysates of WT/WT or ex/ ex mice at different time points after ischemia $(n=4)$. (D-G) Macrophage and neutrophil infiltration in the kidney of ADAM17ex/ex mice ( $\mathbf{D}$ and $\mathbf{E} ; n=5-6$ ) or PTC-KO (Cre fl/fl) mice (F and $\mathbf{G} ; n=4-5$ ) and their WT/WT littermates was examined by immunostaining at different time points after ischemia or 7 days after UUO $(n=5) .{ }^{*} P<0.05 ;{ }^{* *} P<0.01 ;{ }^{* * *} P<0.001$ as determined by an unpaired 2-tailed Student's $t$ test. ADAM17, a disintegrin and metalloprotease 17; IRI, ischemia reperfusion injury; MCP1, monocyte chemoattractant protein 1; PDGFR $\beta$, platelet derived growth factor receptor beta; PTC-KO, proximal tubule cell $\mathrm{KO}$; UUO, unilateral ureteral obstruction.

gest that it is the release of EGF ligand ADAM17 substrates that causes EGFR activation. We have only observed low EGFR Y845 phosphorylation in our studies at all time points examined (data not shown). Complicating the interpretation of this issue is the fact that SRC can be activated by EGFR itself, making it difficult to examine whether the action of SRC is upstream or downstream of EGFR activation. Of note, some studies have implicated ROS (35) and also SRC in the activation of ADAM17 (36). Taking all evidence into account, it is thus possible that Y 845 phosphorylation by SRC represents an additional modulatory pathway to sustain ligand-dependent EGFR activation after injury.

Since all major cell types in the injured kidney can produce or respond to elevated amounts of EGFR ligands or $\mathrm{TNF} \alpha(6,37)$, analysis of their cause and effect in kidney injury and fibrosis has been complicated. Our ADAM17 PTC-KO clearly shows that the PTC is responsible for sending an injurious profibrotic signal (chronic EGFR ligand and TNF $\alpha$ release; proinflammatory cytokine and TGF $\beta$ production) to tubular cells, stromal cells, neutrophils/macrophages, and possibly endothelial cells. ADAM17 stromal cell KO (FoxD1-Cre) was not protective against fibrosis (at least in UUO), suggesting that ADAM17 substrate release from fibroblasts does not play a dominant profibrotic role. Thus, stromal cells and (myo)fibroblasts derived from them act as downstream effectors of ADAM17-induced profibrotic signals emanating from injured PTC. Our data does not exclude possible profibrotic ADAM17 functions in other kidney cells, but the significant reduction in fibrosis afforded by proximal tubule deletion highlights this segment of the nephron as the most important.

Our results strongly implicate interplay of both physiological ADAM17 downstream pathways - the EGFR pathway, with strong evidence for a role of AREG, and the TNF $\alpha$ pathway - in establishing sustained tubular EGFR activation and fibrosis. AREG was by far the most upregulated EGFR ligand in all our kidney injury models and also in human kidney disease samples. AREG has also been found upregulated in mouse lung and liver injury models and in patients with idiopathic pulmonary fibrosis or chronic liver injury with fibrosis (38-40). Compared with any other EGFR ligand tested or with TNF $\alpha$, AREG was uniquely able to upregulate itself (Figure 5 and Supplemental Figure 4) and all ADAM17 pathway components, thereby strengthening its own signal; it also caused the most sustained EGFR activation and TGF $\beta$ 


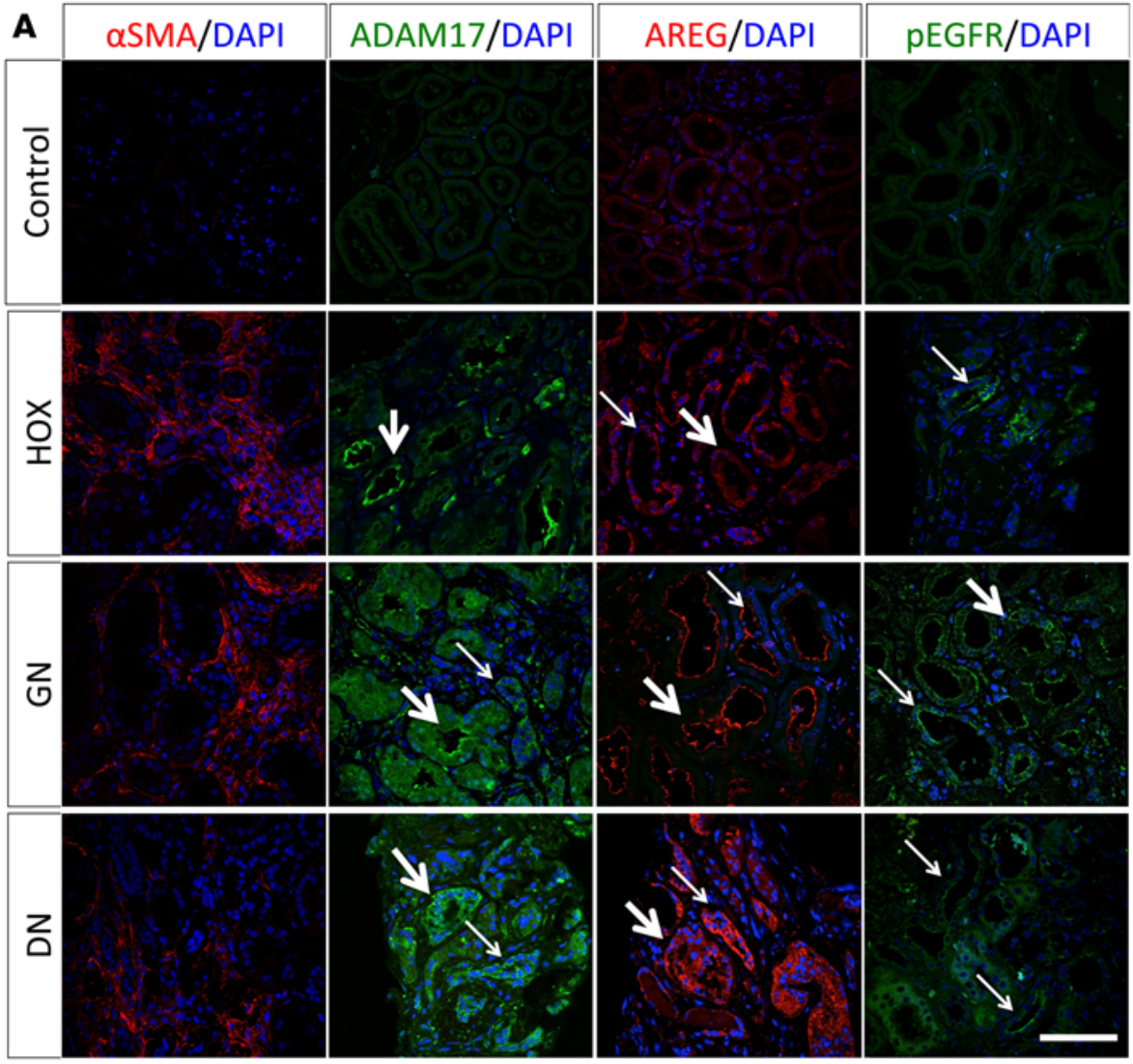

B

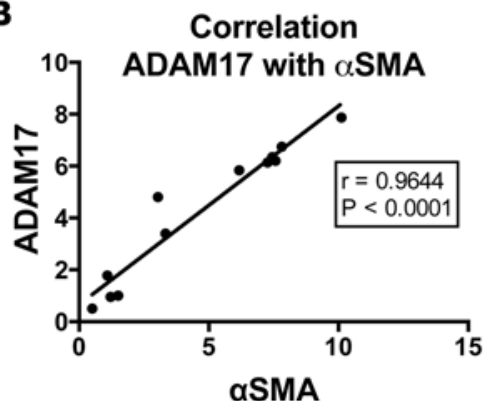

C

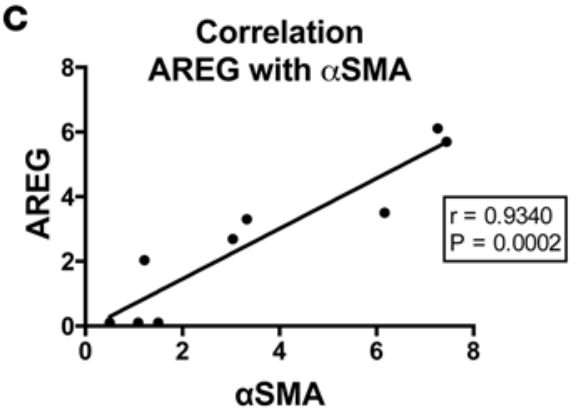

Figure 7. Induction of ADAM17 and AREG protein expression and EGFR phosphorylation in human kidney disease. (A) The presence of the fibrotic marker $\alpha$ SMA and of ADAM17, AREG, and phospho-EGFR were examined in human kidney biopsies by immunostaining. Representative images of hyperoxaluria (HOX; intrarenal tubular obstruction by crystals not shown in image), glomerulonephritis (GN), and diabetic nephropathy (DN) cases are shown (4 controls, $4 \mathrm{HOX}, 4 \mathrm{GN}$, and 4 diabetic patients were examined; representative images are shown) (thick arrows: positive staining in proximal tubular-like structures; thin arrows: positive staining in distal tubular-like structures; scale bar: $50 \mu \mathrm{m}$ ). (B and C) Correlation of ADAM17 (B) or AREC (C) protein expression levels with $\alpha$ SMA levels across all patients studied $(n=9-12)$. The Pearson correlation coefficient $(R)$ and $P$ value are provided in each graph. ADAM17; a disintegrin and metalloprotease 17; AREG, amphiregulin; EGFR, epidermal growth factor receptor; $\alpha \mathrm{SMA}$, alpha smooth muscle actin.

and inflammatory cytokine production in proximal tubule cells. Upregulation of the AREG promoter by AREG itself (in a positive-feedback loop) or by other members of the EGF family has been reported (41-43). The addition of TNF $\alpha$ strongly enhanced AREG's ability to induce TGF $\beta$ and inflammatory cytokines in our in vitro experiments in PTC, suggesting strong synergy of both ADAM17 pathways. The nature of the molecular mechanisms of TNF $\alpha$-AREG crosstalk will need to be studied in detail but could involve TNF $\alpha$ induction of AREG cleavage itself (Figure 5C), as was also reported (e.g., in hepatocytes; ref. 44) or the cross-activation of similar downstream pathways. Of note, at the receptor level soluble AREG acts as a low-affinity EGFR ligand (45), which — in contrast to the higher-affinity ligands EGF or HB-EGF — does not lead to significant downregulation of the receptor from the cell surface (46-48). This additional feature might distinguish profibrotic from prorepair EGFR ligands and enhance sustained EGFR activation, as shown in our experiments with AREG in PTC.

$\mathrm{TNF} \alpha$ cleavage in the kidney in vivo was significantly reduced in ADAM17ex/ex mice and correlated with a strong reduction in kidney proinflammatory markers and kidney ingress of macrophages and neutrophils after injury. In ADAM17 PTC-KO mice, we also detected significant reductions in macrophage and neutrophil ingress into the kidney after injury. Macrophage ingress showed only a nonsignificant trend toward reduction as compared with WT littermates after IRI, but it was significantly reduced after UUO (Figure 6F). Neutrophils were significantly reduced after IRI (Figure 6G). It is possible that the observed differences in macrophage ingress after IRI between ADAM17ex/ex and ADAM17 PTC-KO mice depend in part on differences in mouse background and timing of examination. However, together with the in vitro results obtained in PTC, our overall results in vivo suggest that the proximal tubule represents a significant source of ADAM17-dependent inflammatory signals, such as TNF $\alpha$ and MCP1, that attract macrophages and neutrophils into the kidney. Decreased macrophage ingress likely contributes to decreased TNF $\alpha$ expression/cleavage in ADAM17 hypomorphic or ADAM17 PTC-KO mice, since macrophages express 
A

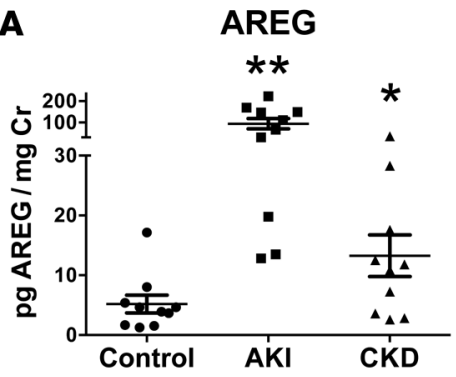

C

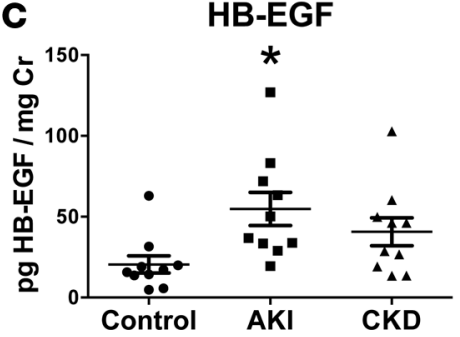

E

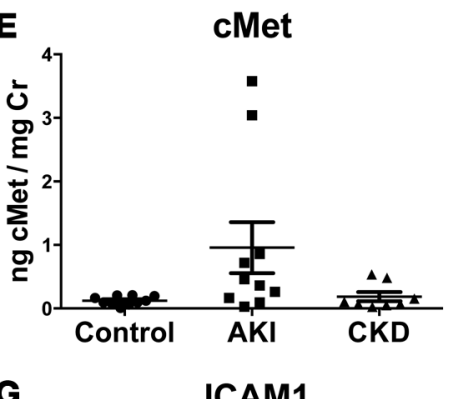

G

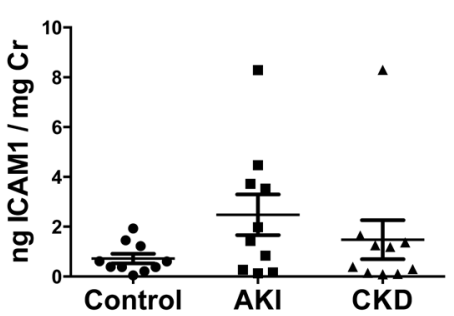

B
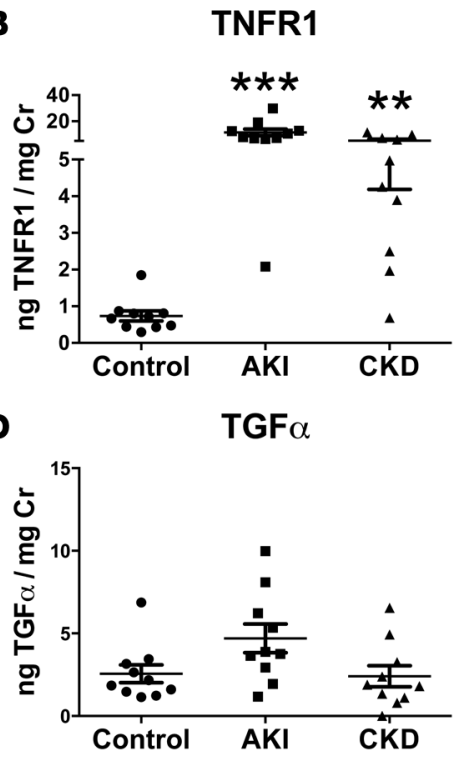

$\mathbf{F}$
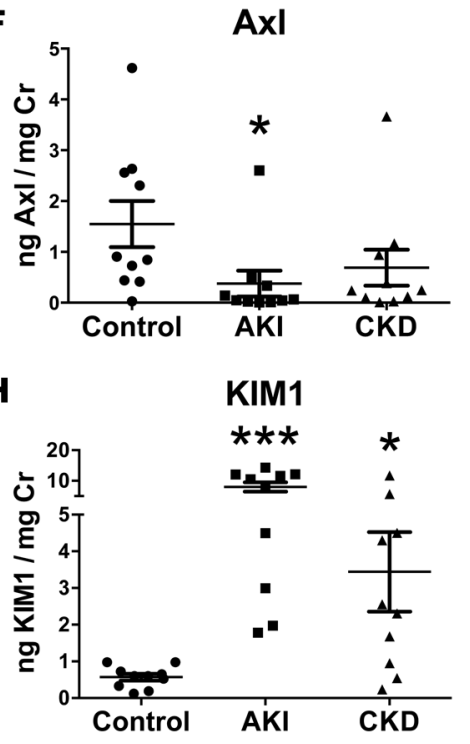

Figure 8. Soluble AREG and TNFR1 are significantly increased in the urine of AKI and CKD patients. The urinary levels of the soluble ADAM17 substrates (AREG [A], TNFR1 [B], HB-EGF [C], TGF $\alpha$ [D], AxI [F], ICAM1 [C], and KIM1 [H]) and of the ADAM10 substrate CMET (E) were measured by ELISA in control, AKI, and CKD patients ( $n=10 ; 50 \%$ of CKD patients were diabetics; patient demographics and details in Supplemental Table 1). ${ }^{*} P<0.05 ;{ }^{*} P<0.01 ;{ }^{* *} P<0.001$ as determined by an unpaired 2-tailed Student's $t$ test. AREG, amphiregulin; TNFR, tumor necrosis factor receptor; AKI, acute kidney injury; CKD, chronic kidney disease; ADAM17, a disintegrin and metalloprotease 17; HB-EGF, heparin binding epidermal growth factor-like growth factor; ICAM1, intercellular adhesion molecule 1; KIM1, kidney injury molecule 1; cMET, met.

ADAM17 and represent a major source of $\mathrm{TNF} \alpha$. Depletion of macrophages before ischemia/reperfusion indeed diminishes kidney injury; however, the role of macrophages in AKI is complex, as they can not only play injurious roles, but are also ultimately needed for kidney repair (49).

Further supporting TNFa's involvement in the inflammatory component of AKI, TNFR1 (TNF $\alpha$ receptor) $\mathrm{KO}$ mice or rats treated with etanercept (TNF $\alpha$ neutralizing antibody) show less tubular injury, inflammation, and neutrophil ingress after ischemic AKI (50, 51). Also, in biopsies of various human fibrotic kidney diseases, ADAM17 expression levels positively correlate with the presence of macrophages (52). Interestingly, TNFR1/2 are also ADAM17 substrates $(32,33)$, and soluble TNFR1/2 in serum of patients is associated with the progression of diabetic kidney disease, which is well-known to be associated with progressive tubulo-interstitial fibrosis $(53,54)$. How cleavage and the appearance of soluble TNFR1/2 is pathogenetically linked to the progression of disease is unknown.

Several other EGFR ligands have also been linked to kidney fibrosis. As examples, chronic angiotensin II infusion in mice caused ADAM17 cleavage of TGF $\alpha$ and sustained EGFR activation in tubular cells and kidney fibrosis, while TGF $\alpha$ KO mice were protected (22). We did not find significant upregulation of TGF $\alpha$ in our rather different injury models. In mice, transgenic overexpression of HB-EGF in the heart or pancreas $(55,56)$ and of TGF $\alpha$ in the lung (57) causes fibrosis. Involvement of specific EGFR ligands could occur due to organ-specific expression context or due to a specific EGFR ligand feature, such as those we described for AREG. It is, however, possible that nonphysiologically regulated chronic EGFR ligand overexpression or exposure (induction by chronic angiotensin II infusion) causes enough sustained EGFR activation to induce fibrosis, independent of the ligand in question. Of note, ADAM17 has been shown to cleave many substrates in cell lines in vitro (58), raising the possibility that other ADAM17 substrates might be involved. However, based on observations in humans and mice, EGF ligands and TNF $\alpha$ and likely IL6 receptor (IL-6R) indeed represent ADAM17's most important physiologically relevant substrates in vivo $(16,17,59)$. Trans-signaling of soluble IL-6R has been linked to crescentic glomerulonephritis $(60,61)$.

Our results in human tissues and urines of patients with $\mathrm{AKI}, \mathrm{CKD}$, or urinary obstruction suggest that ADAM17 pathways, EGFR and TNF $\alpha$, are also involved in various human fibrotic kidney diseases. In human biopsy studies, ADAM17 expression was found significantly upregulated in transplanted kidneys with interstitial fibrosis-tubular atrophy (IF-TA) (62) and in various chronic fibrotic kidney diseases, including diabetic nephropathy, and many forms of glomerular disease (52). In the latter study ADAM17 


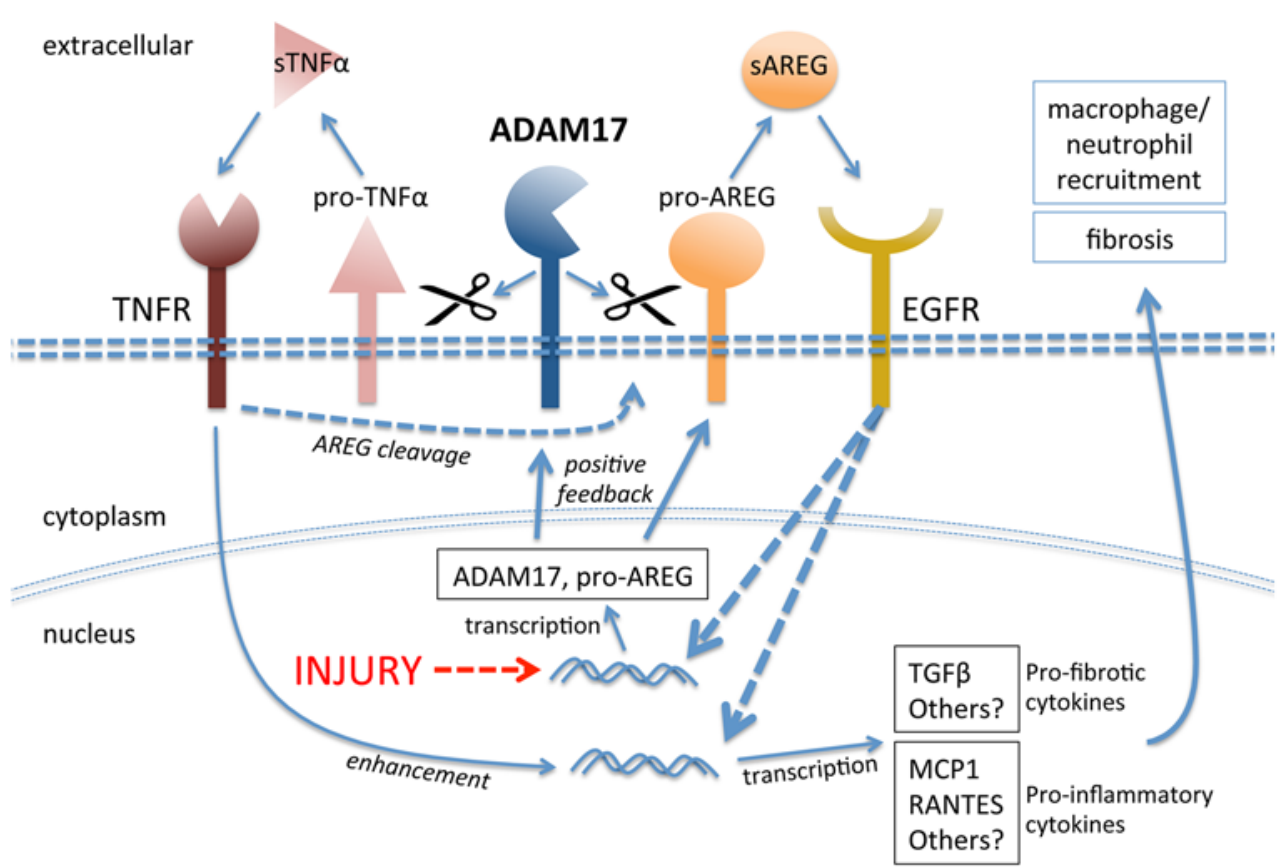

Figure 9. Mechanisms of injury-induced ADAM17-dependent sustained EGFR activation in proximal tubule cells. Injury drives upregulation of ADAM17 and of its substrates, in particular pro-AREG. Increased ADAM17 activity on the cell surface leads to enhanced release of soluble AREG and TNF $\alpha$, which activate their respective receptors. In a positive feedback loop, AREG-mediated EGFR activation increases ADAM17 and AREG expression. TNF $\alpha$-mediated TNFR activation strengthens this feedback loop further by enhancing soluble AREG release (pathway crosstalk). Both pathways, AREG/EGFR and TNF $\alpha / T N F R$, potentiate each other in inducing profibrotic and proinflammatory cytokines that drive kidney fibrosis. pro-TNF $\alpha$, transmembrane pro-tumor necrosis factor $\alpha$; sTNF $\alpha$, soluble tumor necrosis factor $\alpha$; ADAM17, a disintegrin and metalloprotease 17; EGFR, epidermal growth factor receptor; pro-AREG, proamphiregulin; SAREG, soluble amphiregulin; TNFR, tumor necrosis factor receptor; MCP1, monocyte chemoattractant protein 1.

expression levels in the proximal tubule correlated positively with the extent of macrophage ingress and interstitial fibrosis present in the sample and correlated negatively with glomerular filtration rate (a measure of kidney function). Taken together, these results suggest that the ADAM17 pathway is also active in human kidney disease, and its activity is positively correlated with functional decline. Whether AREG could serve as a biomarker for the progression of CKD in AKI, CKD or other fibrotic kidney diseases will require further studies. Recently, the presence of soluble EGF, an EGF ligand released by ADAM10, in the urine of a mixed group of CKD patients was found to be positively correlated with preservation of estimated glomerular filtration rate and kidney function (41). We observed a strong decline of EGF levels in our acute injury models, and they stayed low throughout injury/repair up to at least 42 days. It is possible that levels of soluble EGF in the urine in general reflect tubular epithelial cell health, correlate with the number of intact cells and tubules, and decline with fibrosis.

In summary, our results strongly support the notion that the ADAM17-induced pathways AREG-EGFR and TNF $\alpha$-TNFR1/2 act together to mediate kidney fibrosis after injury and identify ADAM17 and its dependent pathways as possible new targets in the prevention or treatment of human fibrotic kidney disease. Targeting of the ADAM17 axis in kidney disease in humans could be tested with existing drugs for the specific inhibition of EGFR or TNF $\alpha$ used in other human diseases, and these drugs could then possibly be repurposed for applications in kidney disease. Specific contributions of EGFR and TNF $\alpha$ pathways need to be explored further (e.g., by using specific pharmacological inhibitors, alone or in combination, and by creating ADAM17 substrate KOs in tubular or other kidney cells).

\section{Methods}

Animal experiments. ADAM17ex/ex have been described (17). SLC34a1-GCE mice and FoxD1-GC mice have been described previously $(14,29)$. ADAM17 ${ }^{\mathrm{fl} / \mathrm{fl}}$ (or TACE ${ }^{\text {flox }}$ ) mice were obtained from The Jackson Laboratory, strain 009597. We bred the Cre and flox lines together for 2 generations and then selected 
breeders to produce the experimental genotypes $\mathrm{ADAM} 17^{\mathrm{WT} / \mathrm{WT}} \mathrm{Cre}^{+/-}$mice (control group) and ADAM$17^{\mathrm{f} / \mathrm{fl}} \mathrm{Cre}^{+/-}$(ADAM17 PTC-KO when using SLC34a1-GCE; stroma-KO when using FoxD1-GC). For induction of the SLC34a1-driven Cre, mice were injected with 3 doses of tamoxifen $10 \mathrm{mg}$ (every other day), and surgery was only performed 1 week later to allow for tamoxifen washout. For all studies adult (8- to 12-week-old) male mice were used in accordance with the animal care and use protocol approved by the Institutional Animal Care and Use Committee of Harvard Medical School.

Ischemia for 30 minutes (ADAM17ex/ex and WT littermates) or 29 minutes (ADAM17 PTC-KO and WT littermates) at $37^{\circ} \mathrm{C}$ was induced in both kidneys using the flank approach as previously reported (63). Sham operations were performed with exposure of both kidneys but without induction of ischemia. For inhibitor experiments $0.5 \mathrm{mg} / \mathrm{kg}$ of ADAM17 prodomain inhibitor (28) or vehicle $(0.25$ $\mathrm{ml}$ PBS) was injected i.p. daily.

UUO was executed as described previously (63). Briefly, after flank incision, the left ureter was tied off at the level of the lower pole with two 3.0 silk ties. Sham-operated mice underwent the same surgical procedure, except for the ureter ligation. Seven or 14 days following surgery, mice were sacrificed, and the obstructed and contralateral nonobstructed kidneys were harvested for analysis.

Renal function and histology. Serum creatinine was assessed by HPLC (64) at the O'Brien Core Center for Acute Kidney Injury Research (University of Alabama School of Medicine, Birmingham, Alabama, USA). BUN levels were measured using the Infinity Urea assay (Thermo Scientific) according to the manufacturer's instructions. KIM1 and NGAL were measured by using Luminex xMAP Technology, as previously described (65).

Kidney histology was examined in formalin-fixed H\&E, PAS, or Masson's trichrome-stained sections. The degree of tubulointerstitial damage was scored semiquantitatively (scale 1-5) as previously described (66). Fibrosis severity was scored in kidney cortex at $\times 400$ magnification using a counting grid with 144 intersections. The number of grid intersections overlying trichrome-positive (blue) interstitial areas was counted and expressed as a percentage of all grid intersections. For this calculation, intersections that were in tubular lumen and glomeruli were subtracted from the total number of grid intersections. The Masson's trichrome-stained sections were also used for the scoring of the cortical interstitial area and tubular dilation. Of note, we used representative images of cortical areas throughout this manuscript for all immunofluorescence stains shown.

Immunofluorescence staining of the kidney was performed on paraffin or frozen sections following standard protocols. The primary Abs included: ADAM17 (a gift from Carl Blobel, Hospital for Special Surgery, New York, New York, USA), aSMA (CY3-conjugated, Sigma-Aldrich, C6198), fibronectin (Abcam, ab2413), CD31 (eBioscience, 14-0311) pEGFR Y1068 (Cell Signaling Technology, 3777), pEGFR Y1173 (R\&D Systems, AF1095), and AREG (R\&D Systems, BAF262). Fluorescein-LTL was from Vector Laboratories. Images were analyzed using ImageJ (67).

Cell culture and treatments. Primary cultures of kidney tubular cells from ADAM17ex/ex or WT litteremates were generated using established methods (68). Cells were used in experiments between days 5 and 7 of culture. A human proximal tubular cell line (HPTC-05; ref. 69) was also used. All tubular cells were maintained in DMEM/F12 medium supplemented with $10 \mathrm{ng} / \mathrm{ml}$ EGF, $0.05 \mathrm{mM}$ L-ascorbic-2 phosphate, $0.004 \mathrm{mg} / \mathrm{ml}$ dexamethasone, $1.5 \mathrm{mM} \mathrm{HEPES}, 1.2 \mathrm{mg} / \mathrm{ml} \mathrm{NaHCO}_{3}$, and 5\% FBS. Fibroblast were grown in DMEM with $10 \%$ FBS.

All experiments were performed using subconfluent cells that were serum deprived overnight. TNF $\alpha$ $(10 \mathrm{ng} / \mathrm{ml})$ and/or equimolar $(17 \mathrm{pM})$ amounts of the different EGFR ligands (all from R\&D Systems) were added to the cells for different time points as noted. When inhibitors were used, cells were pretreated with the respective inhibitor for 30 minutes before the addition of the ligands.

Quantitative PCR. Total RNA was isolated from either the kidneys or cultured cells using the QIAzol reagent (Qiagen) according to the standard protocol, and total RNA was reverse transcribed using the QuantiTect RT kit (Qiagen). Real-time PCR was performed with the Fast SYBR Green (Qiagen) in a 384well plate format using the QuantStudio 7 Flex system (Applied Biosystems). GAPDH or RPLP0 were used as housekeeping genes. Data were analyzed using the $\Delta \Delta \mathrm{Ct}$ method. Primer sequences are provided in Supplemental Table 2.

Protein sample preparation and Western blot. Whole kidney or cell lysates were prepared as previously described and analyzed by standard Western blot techniques. The primary antibodies used include ADAM17, fibronectin (Abcam, ab2413), pEGFR Y1068 (Cell Signaling Technology, 2234 and 3777), 
pERK1/2 (Cell Signaling Technology, 9101), GAPDH (Abcam, ab181602), and tubulin (Abcam, ab7291).

ELISA. Mouse TNF $\alpha$ and AREG were measured in kidney lysates using ELISA kits (900-M54 PeproTech and DY989 R\&D Systems) according to the manufacturer's instructions. Human TGF $\alpha$, HB-EGF, AREG, TNFR1, ICAM1, Axl, and cMET were measured in human urine samples using microsphere-based Luminex Technology and ELISA kits (DY239, DY259, DY262, DY225, DY720, DY154, DY358, respectively; all from R\&D Systems). Protein levels were calculated after correction for urine creatinine as described (65).

Statistics. All results are reported as the mean \pm SD. Comparison of 2 groups was performed using an unpaired, 2-tailed $t$ test or a Pearson correlation analysis where appropriate. Statistical analyses were performed using GraphPad Prism, version 6.0 (GraphPad Software Inc.). A $P$ value of less than 0.05 was considered significant.

Study approval. Use of mice in this study was reviewed and approved by the Standing Committee on Animals at Harvard Medical School, in adherence to standards set in the Guide for the Care and Use of Laboratory Animals (8th edition, The National Academies Press, revised 2011). Specimens for human study analysis were taken from patients after informed consent. The use of these specimens was approved by the IRB of the Brigham and Women's Hospital, in adherence to the Declaration of Helsinki.

\section{Author contributions}

$\mathrm{AH}$ conceived and coordinated the study and wrote the paper. EK designed, performed, and analyzed all the data and contributed to writing the paper. MLM and JK performed part of the experiments and analyzed part of the data. XS performed animal surgeries. VS provided expertise for ELISA multiplex assays. AC and SRJ provided ADAM17ex/ex mice. EW and IS developed and provided the ADAM17 prodomain inhibitor. SSW provided urinary samples, and HR provided human kidney biopsy samples. BDH provided SLC34A1-Cre and FoxD1-Cre mice and expertise for analyzing fibrotic kidneys. JVB provided expertise on IRI and UUO models.

\section{Acknowledgments}

We thank Carl Blobel for the provision of ADAM17 antibodies. AH and EK were supported by National Institute of Diabetes and Digestive and Kidney Diseases (NIDDK) R00DK077731 and HSCI DP-0150-15-00.

Address correspondence to: Andreas Herrlich, Division of Nephrology, Washington University School of Medicine, 660 S. Euclid Avenue, Wohl Clinic, Campus Box 8126, St. Louis, Missouri 63110. Phone: 617.525.5914; E-mail: aherrlich@wustl.edu.

1. Parr SK, Siew ED. Delayed consequences of acute kidney injury. Adv Chronic Kidney Dis. 2016;23(3):186-194.

2. Rewa O, Bagshaw SM. Acute kidney injury-epidemiology, outcomes and economics. Nat Rev Nephrol. 2014;10(4):193-207.

3. Declèves AE, Sharma K. Novel targets of antifibrotic and anti-inflammatory treatment in CKD. Nat Rev Nephrol. 2014;10(5):257-267

4. Bonventre JV, Yang L. Cellular pathophysiology of ischemic acute kidney injury. J Clin Invest. 2011;121(11):4210-4221.

5. Grgic I, et al. Targeted proximal tubule injury triggers interstitial fibrosis and glomerulosclerosis. Kidney Int. 2012;82(2):172-183.

6. Liu J, et al. Cell-specific translational profiling in acute kidney injury. J Clin Invest. 2014;124(3):1242-1254.

7. Bonventre JV, Zuk A. Ischemic acute renal failure: an inflammatory disease?. Kidney Int. 2004;66(2):480-485.

8. Chen J, Chen JK, Harris RC. EGF receptor deletion in podocytes attenuates diabetic nephropathy. J Am Soc Nephrol. 2015;26(5):1115-1125.

9. Chen J, et al. EGFR signaling promotes TGF $\beta$-dependent renal fibrosis. J Am Soc Nephrol. 2012;23(2):215-224.

10. Wang Z, Chen JK, Wang SW, Moeckel G, Harris RC. Importance of functional EGF receptors in recovery from acute nephrotoxic injury. J Am Soc Nephrol. 2003;14(12):3147-3154.

11. Tang J, Liu N, Zhuang S. Role of epidermal growth factor receptor in acute and chronic kidney injury. Kidney Int. 2013;83(5):804-810.

12. Tang J, et al. Sustained activation of EGFR triggers renal fibrogenesis after acute kidney injury. Am J Pathol. 2013;183(1):160-172.

13. Liu N, et al. Genetic or pharmacologic blockade of EGFR inhibits renal fibrosis. J Am Soc Nephrol. 2012;23(5):854-867.

14. Humphreys BD, et al. Fate tracing reveals the pericyte and not epithelial origin of myofibroblasts in kidney fibrosis. Am J Pathol. 2010;176(1):85-97.

15. Gooz M. ADAM-17: the enzyme that does it all. Crit Rev Biochem Mol Biol. 2010;45(2):146-169.

16. Blaydon DC, et al. Inflammatory skin and bowel disease linked to ADAM17 deletion. N Engl J Med. 2011;365(16):1502-1508.

17. Chalaris A, et al. Critical role of the disintegrin metalloprotease ADAM17 for intestinal inflammation and regeneration in mice. JExp Med. 2010;207(8):1617-1624.

18. Humes HD, Cieslinski DA, Coimbra TM, Messana JM, Galvao C. Epidermal growth factor enhances renal tubule cell regeneration 
and repair and accelerates the recovery of renal function in postischemic acute renal failure. J Clin Invest. 1989;84(6):1757-1761.

19. Zhuang S, Yan Y, Daubert RA, Schnellmann RG. Epiregulin promotes proliferation and migration of renal proximal tubular cells. Am J Physiol Renal Physiol. 2007;293(1):F219-F226.

20. Zhuang S, Dang Y, Schnellmann RG. Requirement of the epidermal growth factor receptor in renal epithelial cell proliferation and migration. Am J Physiol Renal Physiol. 2004;287(3):F365-F372.

21. Laouari D, et al. A transcriptional network underlies susceptibility to kidney disease progression. EMBO Mol Med 2012;4(8):825-839.

22. Lautrette A, et al. Angiotensin II and EGF receptor cross-talk in chronic kidney diseases: a new therapeutic approach. Nat Med. 2005;11(8):867-874.

23. Bollée G, et al. Epidermal growth factor receptor promotes glomerular injury and renal failure in rapidly progressive crescentic glomerulonephritis. Nat Med. 2011;17(10):1242-1250

24. Sanchez-Niño MD, et al. TNF superfamily: a growing saga of kidney injury modulators. Mediators Inflamm. 2010;2010:182958.

25. Morimoto Y, et al. TNF-alpha deficiency accelerates renal tubular interstitial fibrosis in the late stage of ureteral obstruction. Exp Mol Pathol. 2008;85(3):207-213.

26. Guo G, Morrissey J, McCracken R, Tolley T, Liapis H, Klahr S. Contributions of angiotensin II and tumor necrosis factor-alpha to the development of renal fibrosis. Am J Physiol Renal Physiol. 2001;280(5):F777-F785.

27. Wong E, Maretzky T, Peleg Y, Blobel CP, Sagi I. The Functional Maturation of A Disintegrin and Metalloproteinase (ADAM) 9, 10, and 17 requires processing at a newly identified proprotein convertase (PC) cleavage site. J Biol Chem. 2015;290(19):12135-12146.

28. Sagi I, Wong E, Afik R, Research Y, inventors; Yeda Research Development Co. LTD, assignee. Variants of tace pro-domain as TNF-a inhibitor their medical use. International patent no. PCT/IL2013/050400. International Filing Date: September 5, 2013.

29. Kusaba T, Lalli M, Kramann R, Kobayashi A, Humphreys BD. Differentiated kidney epithelial cells repair injured proximal tubule. Proc Natl Acad Sci U S A. 2014;111(4):1527-1532.

30. Coca SG, Singanamala S, Parikh CR. Chronic kidney disease after acute kidney injury: a systematic review and meta-analysis. Kidney Int. 2012;81(5):442-448.

31. Chawla LS, Kimmel PL. Acute kidney injury and chronic kidney disease: an integrated clinical syndrome. Kidney Int. 2012;82(5):516-524.

32. McMahan RS, Riehle KJ, Fausto N, Campbell JS. A disintegrin and metalloproteinase 17 regulates TNF and TNFR1 levels in inflammation and liver regeneration in mice. Am J Physiol Gastrointest Liver Physiol. 2013;305(1):G25-G34.

33. Maney SK, et al. Deletions in the cytoplasmic domain of iRhom 1 and iRhom 2 promote shedding of the TNF receptor by the protease ADAM17. Sci Signal. 2015;8(401):ra109.

34. Chen J, Chen JK, Harris RC. Angiotensin II induces epithelial-to-mesenchymal transition in renal epithelial cells through reactive oxygen species/Src/caveolin-mediated activation of an epidermal growth factor receptor-extracellular signal-regulated kinase signaling pathway. Mol Cell Biol. 2012;32(5):981-991.

35. Willems SH, et al. Thiol isomerases negatively regulate the cellular shedding activity of ADAM17. Biochem J. 2010;428(3):439-450

36. Maretzky T, Zhou W, Huang XY, Blobel CP. A transforming Src mutant increases the bioavailability of EGFR ligands via stimulation of the cell-surface metalloproteinase ADAM17. Oncogene. 2011;30(5):611-618.

37. Grgic I, et al. Translational profiles of medullary myofibroblasts during kidney fibrosis. J Am Soc Nephrol. 2014;25(9):1979-1990.

38. Zhou Y, et al. Amphiregulin, an epidermal growth factor receptor ligand, plays an essential role in the pathogenesis of transforming growth factor- $\beta$-induced pulmonary fibrosis. J Biol Chem. 2012;287(50):41991-42000.

39. Perugorria MJ, et al. The epidermal growth factor receptor ligand amphiregulin participates in the development of mouse liver fibrosis. Hepatology. 2008;48(4):1251-1261.

40. Berasain C, et al. Amphiregulin: an early trigger of liver regeneration in mice. Gastroenterology. 2005;128(2):424-432

41. Willmarth NE, Ethier SP. Autocrine and juxtacrine effects of amphiregulin on the proliferative, invasive, and migratory properties of normal and neoplastic human mammary epithelial cells. J Biol Chem. 2006;281(49):37728-37737.

42. Streicher KL, Willmarth NE, Garcia J, Boerner JL, Dewey TG, Ethier SP. Activation of a nuclear factor $\kappa \mathrm{B} /$ interleukin-1 positive feedback loop by amphiregulin in human breast cancer cells. Mol Cancer Res. 2007;5(8):847-861.

43. Barnard JA, et al. Auto- and cross-induction within the mammalian epidermal growth factor-related peptide family. $J$ Biol Chem 1994;269(36):22817-22822.

44. Berasain C, et al. Epidermal growth factor receptor signaling in hepatocellular carcinoma: inflammatory activation and a new intracellular regulatory mechanism. Dig Dis. 2012;30(5):524-531.

45. Jones JT, Akita RW, Sliwkowski MX. Binding specificities and affinities of egf domains for ErbB receptors. FEBS Lett. 1999;447(2-3):227-231.

46. Stern KA, Place TL, Lill NL. EGF and amphiregulin differentially regulate Cbl recruitment to endosomes and EGF receptor fate. Biochem J. 2008;410(3):585-594

47. Baldys A, et al. Essential role of $\mathrm{c}-\mathrm{Cbl}$ in amphiregulin-induced recycling and signaling of the endogenous epidermal growth factor receptor. Biochemistry. 2009;48(7):1462-1473

48. Roepstorff K, et al. Differential effects of EGFR ligands on endocytic sorting of the receptor. Traffic. 2009;10(8):1115-1127.

49. Lee S, et al. Distinct macrophage phenotypes contribute to kidney injury and repair. J Am Soc Nephrol. 2011;22(2):317-326.

50. Di Paola R, Genovese T, Impellizzeri D, Ahmad A, Cuzzocrea S, Esposito E. The renal injury and inflammation caused by ischemia-reperfusion are reduced by genetic inhibition of TNF- $\alpha \mathrm{R} 1$ : a comparison with infliximab treatment. Eur J Pharmacol. 2013;700(1-3):134-146.

51. Choi DE, Jeong JY, Lim BJ, Na KR, Shin YT, Lee KW. Pretreatment with the tumor nerosis factor- $\alpha$ blocker etanercept attenuated ischemia-reperfusion renal injury. Transplant Proc. 2009;41(9):3590-3596.

52. Melenhorst WB, Visser L, Timmer A, van den Heuvel MC, Stegeman CA, van Goor H. ADAM17 upregulation in human renal disease: a role in modulating TGF- $\alpha$ availability?. Am J Physiol Renal Physiol. 2009;297(3):F781-F790.

53. Niewczas MA, et al. Circulating TNF receptors 1 and 2 predict ESRD in type 2 diabetes. J Am Soc Nephrol. 2012;23(3):507-515.

54. Gohda T, et al. Circulating TNF receptors 1 and 2 predict stage 3 CKD in type 1 diabetes. J Am Soc Nephrol. 2012;23(3):516-524. 
55. Lian H, et al. Heparin-binding EGF-like growth factor induces heart interstitial fibrosis via an Akt/mTor/p70s6k pathway. PLoS One. 2012;7(9):e44946.

56. Means AL, et al. Overexpression of heparin-binding EGF-like growth factor in mouse pancreas results in fibrosis and epithelial metaplasia. Gastroenterology. 2003;124(4):1020-1036.

57. Le Cras TD, et al. Inhibition of PI3K by PX-866 prevents transforming growth factor- $\alpha$-induced pulmonary fibrosis. Am $J$ Pathol. 2010;176(2):679-686

58. Hartmann M, Herrlich A, Herrlich P. Who decides when to cleave an ectodomain?. Trends Biochem Sci. 2013;38(3):111-120.

59. Chalaris A, Garbers C, Rabe B, Rose-John S, Scheller J. The soluble Interleukin 6 receptor: generation and role in inflammation and cancer. Eur J Cell Biol. 2011;90(6-7):484-494.

60. Luig M, et al. Inflammation-induced IL-6 functions as a natural brake on macrophages and limits GN. J Am Soc Nephrol. 2015;26(7):1597-1607.

61. Braun GS, et al. IL-6 trans-signaling drives murine crescentic GN. J Am Soc Nephrol. 2016;27(1):132-142.

62. Mulder GM, et al. ADAM17 up-regulation in renal transplant dysfunction and non-transplant-related renal fibrosis. Nephrol Dial Transplant. 2012;27(5):2114-2122.

63. Yang L, Besschetnova TY, Brooks CR, Shah JV, Bonventre JV. Epithelial cell cycle arrest in G2/M mediates kidney fibrosis after injury. Nat Med. 2010;16(5):535-543.

64. Dunn SR, Qi Z, Bottinger EP, Breyer MD, Sharma K. Utility of endogenous creatinine clearance as a measure of renal function in mice. Kidney Int. 2004;65(5):1959-1967.

65. Sabbisetti VS, Ito K, Wang C, Yang L, Mefferd SC, Bonventre JV. Novel assays for detection of urinary KIM-1 in mouse models of kidney injury. Toxicol Sci. 2013;131(1):13-25.

66. Yang L, et al. KIM-1-mediated phagocytosis reduces acute injury to the kidney. J Clin Invest. 2015;125(4):1620-1636.

67. Schneider CA, Rasband WS, Eliceiri KW. NIH Image to ImageJ: 25 years of image analysis. Nat Methods. 2012;9(7):671-675.

68. Faust J, et al. Correlation of renal tubular epithelial cell-derived interleukin-18 up-regulation with disease activity in MRL-Faslpr mice with autoimmune lupus nephritis. Arthritis Rheum. 2002;46(11):3083-3095.

69. Orosz DE, et al. Growth, immortalization, and differentiation potential of normal adult human proximal tubule cells. In Vitro Cell Dev Biol Anim. 2004;40(1-2):22-34 